\title{
NIRvP: a robust structural proxy for sun-induced chlorophyll fluorescence and photosynthesis across scales
}

Benjamin Dechant ${ }^{1}$, Youngryel Ryu ${ }^{1,2 *}$, Grayson Badgley ${ }^{3}$, Philipp Köhler ${ }^{4}$, Uwe Rascher ${ }^{5}$, Mirco Migliavacca ${ }^{6}$, Yongguang Zhang 7,8 , Giulia Tagliabue ${ }^{9}$, Kaiyu Guan ${ }^{10,11}$, Micol Rossini ${ }^{9}$, Yves Goulas ${ }^{12}$, Yelu Zeng $^{13}$, Christian Frankenberg ${ }^{4,14}$, Joseph A. Berry ${ }^{13}$

${ }^{1}$ Research Institute of Agriculture and Life Sciences, Seoul National University, Seoul, South Korea

${ }^{2}$ Department of Landscape Architecture and Rural Systems Engineering, College of Agriculture and Life Sciences, Seoul National University, South Korea

${ }^{3}$ Black Rock Forest, Cornwall, NY, USA

${ }^{4}$ Division of Geological and Planetary Sciences, California Institute of Technology, Pasadena, CA, USA

5 Institute of Bio- and Geosciences, Plant Sciences (IBG-2), Forschungszentrum Jülich GmbH, 52428 Jülich, Germany

${ }^{6}$ Max Planck Institute for Biogeochemistry, Hans Knoll Straße, 10, D-07745 Jena, Germany

${ }^{7}$ International Institute for Earth System Sciences, Nanjing University, Nanjing, Jiangsu 210023, China.

${ }^{8}$ Jiangsu Provincial Key Laboratory of Geographic Information Technology, Key Laboratory for Land Satellite Remote Sensing Applications of Ministry of Natural Resources, School of Geography and Ocean Science, Nanjing University, Nanjing, Jiangsu 210023, China.

${ }^{9}$ Remote Sensing of Environmental Dynamics Laboratory, Department of Earth and Environmental Sciences (DISAT), University of Milano-Bicocca, Piazza della Scienza 1, Milano, Italy

${ }^{10}$ College of Agricultural, Consumer and Environmental Sciences, University of Illinois at Urbana Champaign, IL, USA

${ }^{11}$ National Center for Supercomputing Applications, University of Illinois at Urbana-Champaign, IL, USA

${ }^{12}$ Dynamic Meteorology Laboratory, Ecole Polytechnique, Palaiseau, France

${ }^{13}$ Department of Global Ecology, Carnegie Institution for Science, Stanford, CA, USA

${ }^{14}$ Jet Propulsion Laboratory, California Institute of Technology, Pasadena, CA, USA

*corresponding author: e-mail address: yryu@snu.ac.kr (Y. Ryu).

Twitter: @ryuyr77

\section{This is a non-peer reviewed preprint submitted to EarthArXiv.}


Sun-induced chlorophyll fluorescence (SIF) is a promising new tool for remotely estimating photosynthesis. However, the degree to which incoming sunlight and the structure of the canopy rather than leaf physiology contribute to SIF variations is still not well characterized. Here we demonstrate that the canopy structure-related near-infrared reflectance of vegetation multiplied by incoming sunlight (NIRvP) is a robust proxy of far-red SIF across a wide range of spatial and temporal scales. Our findings indicate that contributions from leaf physiology to SIF variability are small compared to its structure and radiation components. NIRvP captured spatio-temporal patterns of photosynthesis better than SIF, which seems to be mostly due to the retrieval noise of SIF. Our results highlight the promise of using widely available NIRvP data for vegetation monitoring and also indicate the potential of using SIF and NIRvP in combination to extract physiological information from SIF.

SIF is an electromagnetic signal emitted by chlorophyll $a$ molecules when exposed to sunlight and is increasingly used for vegetation monitoring at regional and global scales ${ }^{1-3}$. SIF is a very weak signal compared to reflected sunlight as only a few percent of the absorbed energy is re-emitted as $\mathrm{SIF}^{3}$. This has important practical implications as the passive retrieval of SIF from the much stronger background signal can result in considerable retrieval noise ${ }^{3-5}$. Along with the photochemical reflectance index ${ }^{6}$, SIF is rather unique among remote sensing indicators in being directly sensitive to changes in leaf photosynthetic physiology $y^{2,7}$. Applications of SIF are diverse including photosynthetic phenology ${ }^{8,9}$, plant stress detection ${ }^{2,10}$, and crop yield estimation ${ }^{11-13}$, but the main goal of most SIF research is the remote estimation of terrestrial gross primary productivity (GPP) $)^{1,14-18}$.

Canopy-level SIF is controlled by three main mechanistic factors, namely the light absorption (absorbed photosynthetically active radiation, APAR), chlorophyll fluorescence emission yield $\left(\Phi_{\mathrm{F}}\right)$, and, in case of far-red SIF at about $760 \mathrm{~nm}$, scattering of emitted fluorescence within the canopy that allows only a fraction of the emitted radiation to escape the canopy ${ }^{19}$ (canopy escape fraction, $\mathrm{f}_{\text {esc }}$; see Eqn. 1 in Methods). While there is considerable knowledge about the dominant role of APAR as driver of SIF ${ }^{14,20,21}$, the separate contributions from $\Phi_{\mathrm{F}}$ and $\mathrm{f}_{\mathrm{esc}}$ to SIF observations are still not well characterized. Several experimental and modelling studies have reported that $\mathrm{f}_{\text {esc }}$ of far-red SIF can vary considerably over time and space due to changes in the canopy structure such as leaf area index, leaf angle distribution and 
clumping ${ }^{18,19,22-24}$. For $\Phi_{\mathrm{F}}$, most work has been conducted at the leaf-level. Although leaf-level $\Phi_{\mathrm{F}}$ responds to environmental stress ${ }^{2,25,26}, \Phi_{\mathrm{F}}$ typically varies relatively little compared to the photochemical quantum yield of photosystem II ( $\Phi_{\text {PSII }}$ ) both at diurnal and seasonal time scales ${ }^{27,28}$. Evergreen needleleaf forests are the exception to this pattern, with considerable seasonal $\Phi_{\mathrm{F}}$ variations due to physiological downregulation of photosynthesis during extended cold periods in winter ${ }^{29,30}$.

Based on current knowledge, canopy-level SIF variations are expected to be driven predominantly by APAR and $f_{\text {esc }}$ rather than $\Phi_{F}$, at least in the absence of strong environmental stress and ecosystems other than evergreen needleleaf forests. At sub-daily time scales, PAR is the dominant driver of SIF given the typically much smaller diurnal variations in the fraction of absorbed PAR (fPAR), $f_{\text {esc }}$ and $\Phi_{F}^{22,27,31}$. At seasonal time scales, APAR and $f_{\text {esc }}$ appear to show stronger variations compared to $\Phi_{\mathrm{F}}^{22,23}$. If indeed APAR

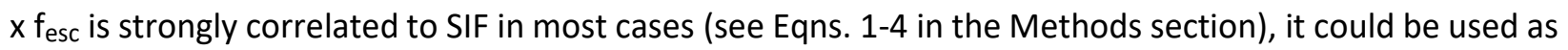
a structural proxy for SIF as recent studies have derived approaches to estimate fPAR $x f_{\text {esc }}$ from widely available reflectance observations ${ }^{19,32}$. In particular APAR $\times f_{\text {esc }}$ can be approximated by $N_{1 R} P^{19,22}$, which is defined as the product of the near infrared reflectance of vegetation $\left(N I R_{V}\right)^{33}$ and incoming PAR (see Methods).

Despite recent findings on canopy structure effects and $\mathrm{f}_{\mathrm{esc}}$ that seem to support the dominance of APAR $\times f_{\text {esc }}$ in variations of $\mathrm{SIF}^{22,32,33}$, a comprehensive evaluation to what degree NIR $\mathrm{P}$ and other related structural SIF proxies explain the variations of canopy SIF has not been reported. In particular, SIF is commonly compared to vegetation indices such as the enhanced vegetation index (EVI $)^{34}$ without considering $\mathrm{PAR}^{33,35,36}$, which does not permit a meaningful direct comparison in cases where spatial and/or temporal variations of PAR are important. Furthermore, previous studies have not evaluated the spatial and temporal patterns of SIF and NIRVP separately, but have instead focused either on long time and large spatial scales ${ }^{33}$ or examined only a few crop sites 22,32 .

Since one of the major goals of SIF research is to improve remote sensing based GPP estimation ${ }^{1,7}$, it is important to also compare the performance of SIF and NIRvP for this purpose. Several recent studies demonstrated the promise of using $\mathrm{NIR}_{V}$ and NIR $\mathrm{P}$ to estimate GPP across multiple spatial scales ${ }^{33,37-39}$. However, direct comparisons of SIF and NIRVP for GPP estimation using observations from the same sensors (or at least from the same viewing geometries) have been limited to a small set of crop sites ${ }^{22,40,41}$. When using observations from different satellites, differences in viewing geometry, overpass time and 
other factors related to data acquisition can affect results in addition to inherent differences between SIF and NIR $P$. Therefore, there is the need to directly compare SIF and NIR $P$ regarding GPP estimation across different ecosystems at the site-level and, using the same satellite sensor for both variables, at the global scale.

In this study, we conducted i) a comprehensive evaluation of the relationship between NIRvP and far-red SIF from plot to global scales and ii) a detailed direct comparison of far-red SIF and NIRVP for GPP estimation with a strong focus on the global scale. We used a unique collection of observations that covers the majority of plant functional types and a wide range of temporal and spatial scales. In particular, this data collection includes observations from tower-based, airborne and satellite instruments with temporal resolutions ranging from half-hourly to monthly and spatial resolutions between $1 \mathrm{~m}$ and $50 \mathrm{~km}$.

\section{Results}

Tower-based measurements. At the site-level, we found very strong linear correlations between SIF and NIRvP at the seasonal time scale (Fig. 1). The squared Pearson correlation $\left(R^{2}\right)$ between SIF and NIR $P$ for individual sites ranged from 0.73 to 0.94 for half-hourly and 0.79 to 0.96 for daily data (Figs. S1a). NIRVP performed slightly better than or similar to FCVIP and EVI2P (see Methods) for individual sites except for the two corn sites, where either FCVIP or EVI2P showed slightly stronger correlations (Fig. S2). NIRvP more clearly outperformed NDVIP, APAR and NIRv alone (Fig. S2). When combining the observations from all sites, the SIF-NIRvP correlation was high $\left(R^{2}=0.86\right.$ and 0.90 for half-hourly and daily data, respectively; Fig. 1 b) and considerably stronger than the APAR-NIRVP correlation $\left(R^{2}=0.74\right.$ for both 30 min and daily data; Fig. 1c). The slopes of the SIF-NIR P relationship differed somewhat between sites (Fig. S1a). Regarding the correlations of SIF and NIR ${ }_{v} P$ to GPP, two previous studies ${ }^{22,40}$ already reported higher or similar correlation of NIR P for the rice, wheat, soybean and the two corn datasets we used. In addition, we found that NIRv P outperformed SIF also for the grassland site $\left(R^{2}=0.60\right.$ vs. 0.49 for half-hourly data; Fig. S1d). 
a)
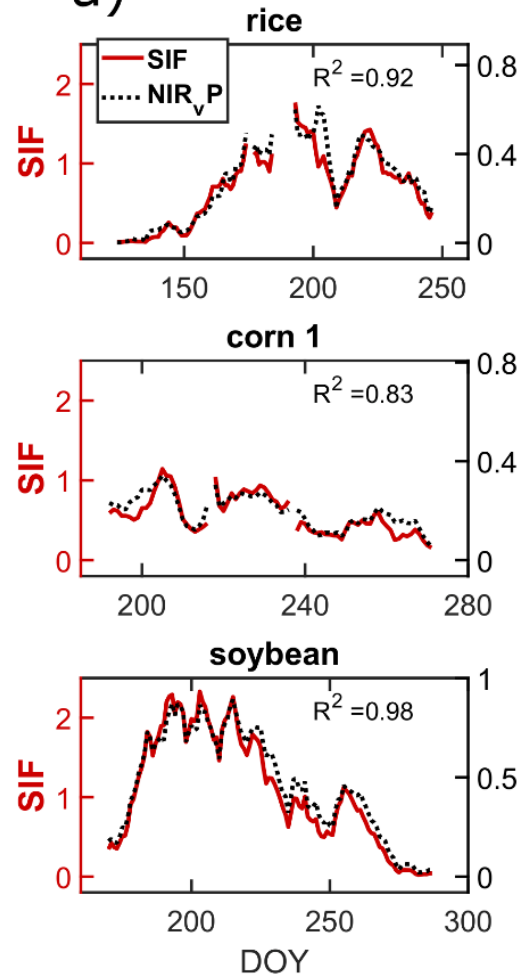
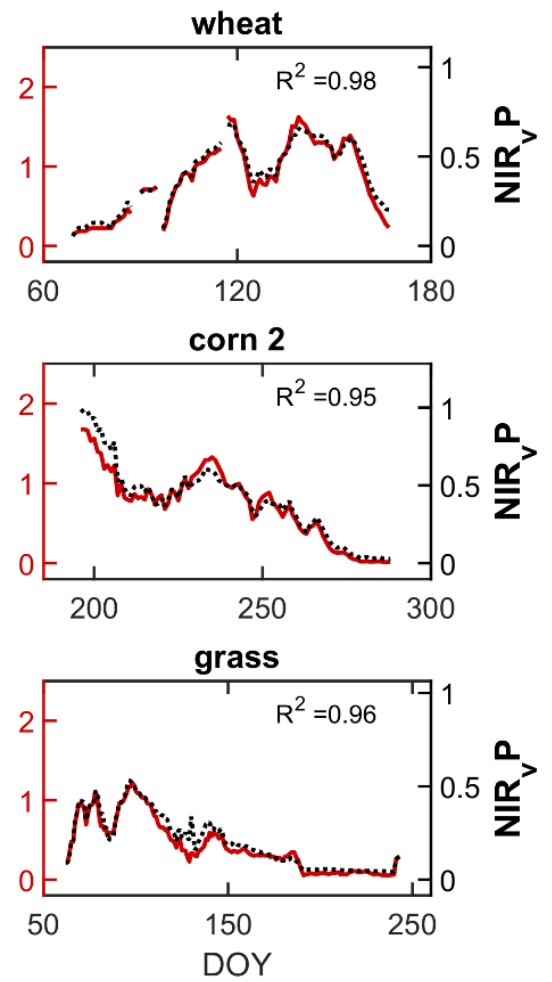

b)
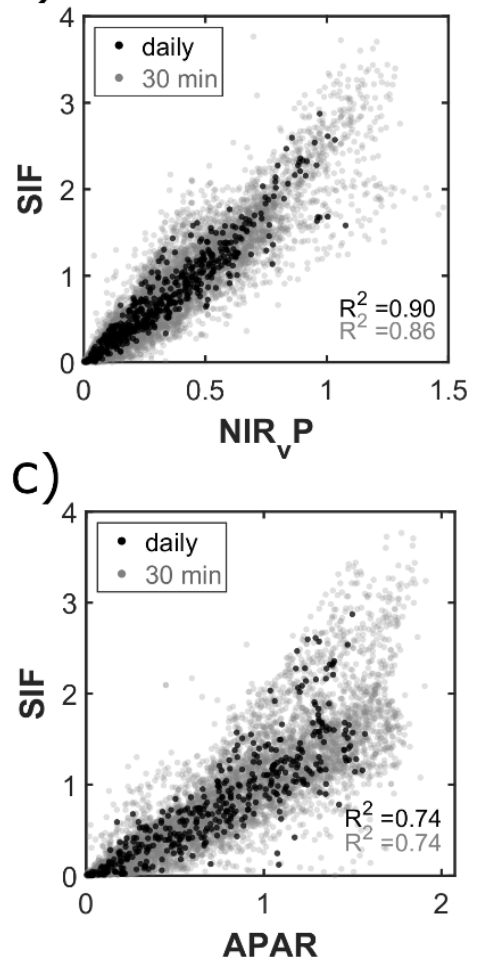

Fig. 1: Temporal dynamics of in-situ SIF and NIRVP and their relationships. Data from six sites distributed in East Asia, Europe and North America are shown. a) Time series of 5-day moving average data are shown, DOY stands for day of the year. b), c) Scatterplot of half-hourly and daily data of SIF vs. NIRVP or in-situ APAR for all site data combined. Note that for C) APAR was not available for the grassland site. SIF is given in units of $m W m^{-2} \mathrm{sr}^{-1} \mathrm{~nm}^{-1}, N I R_{v} P$ and APAR in units of $n m^{-2} \mathrm{~m}^{-2} \mathrm{~s}^{-1}$.

Airborne measurements. At the landscape scale, the SIF-NIRvP relationship was predominantly linear for airborne SIF retrievals at $1 \mathrm{~m}$ resolution in a crop scene $\left(R^{2}=0.86\right)$ and NIRvP captured SIF variations within and between larger crop fields well (Fig. 2a,c). Furthermore, the SIF-NIRvP relationship was strong and linear $\left(R^{2}=0.89\right)$ when selecting a subset of the flight line covering mostly small-scale plots where phenotyping experiments are performed and therefore larger variations in leaf physiology are expected (Figs. 2b,c). We observed a slight tendency towards saturation of NIRvP at high SIF values (Fig. 2a,c). NIRVP had comparable correlation to SIF as FCVI, while EVI2 showed a slightly weaker correlation and NDVI showed strong saturation and only moderate correlation $\left(R^{2}=0.63\right.$; Fig. S2a,b). 
a)
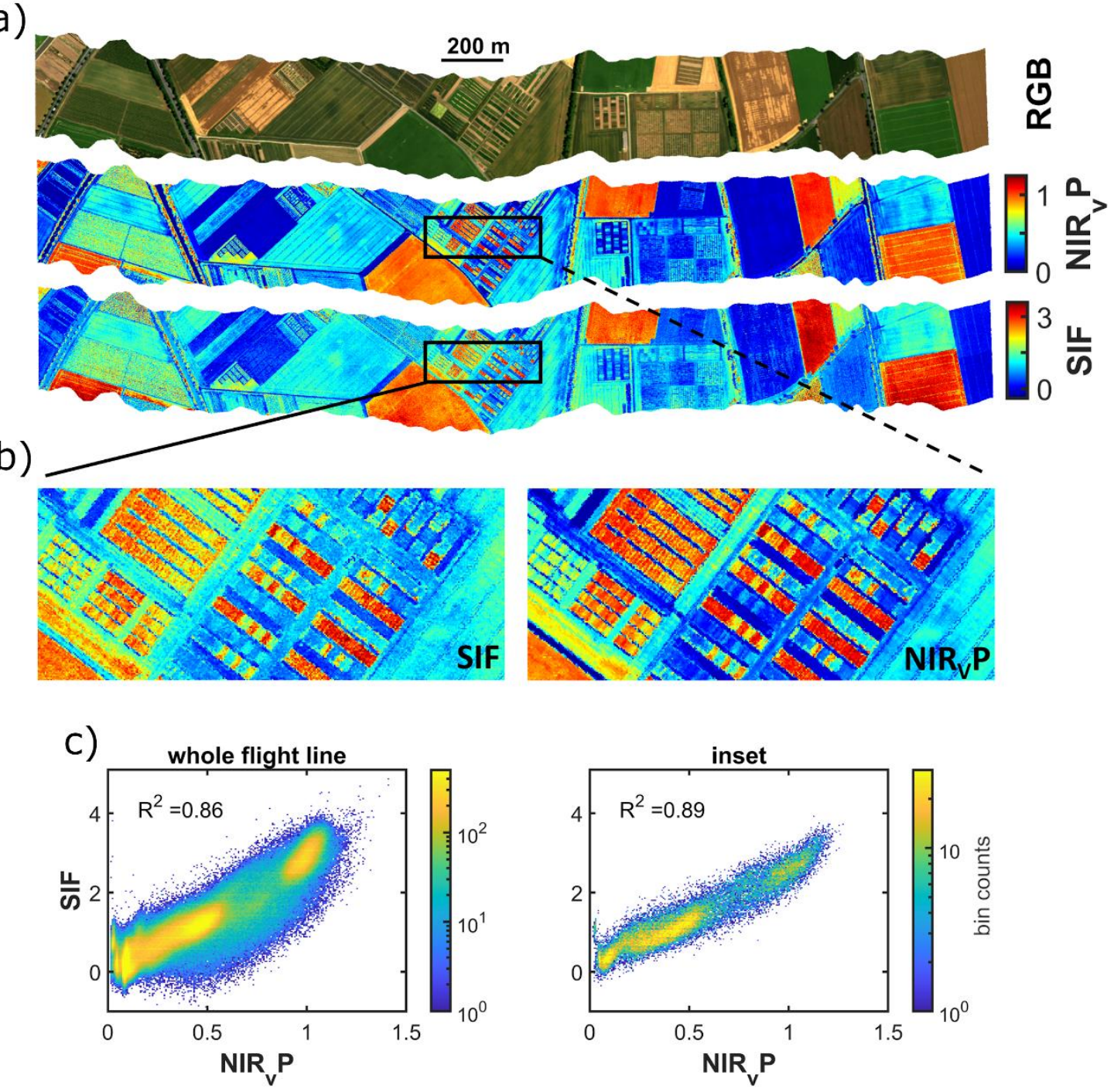

Fig. 2: Spatial patterns and relationships of airborne SIF and NIR P observations over a crop landscape. Data was acquired with the HyPlant sensor over a crop landscape which is part of the agricultural research station Campus Klein-Altendorf, western Germany. All results are based on the original $1 \mathrm{~m}$ spatial resolution data. a) Entire flight lines of SIF, NIRVP and RGB composites, b) zoom to part of the flight line with small phenotyping plots, c) scatterplots corresponding to data shown in a) and b), the color scale indicates bin counts, i.e. point density. SIF is shown in units of $\mathrm{mW} \mathrm{m}^{-2} \mathrm{sr}^{-1} \mathrm{~nm}^{-1}$ and NIRV $P$ in units of $\mathrm{nmol} \mathrm{m}^{-2} \mathrm{~s}^{-1}$. 


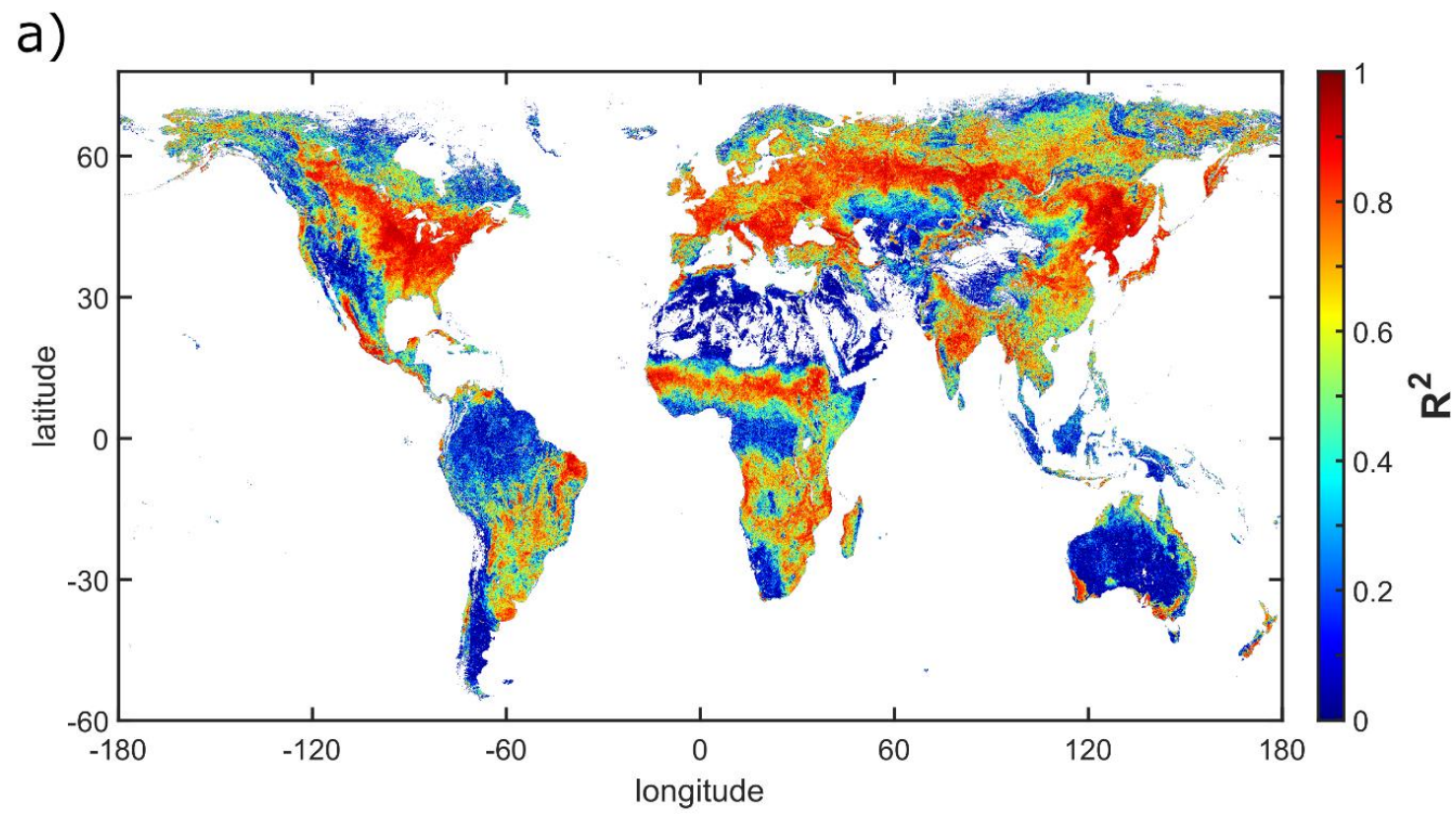

b)
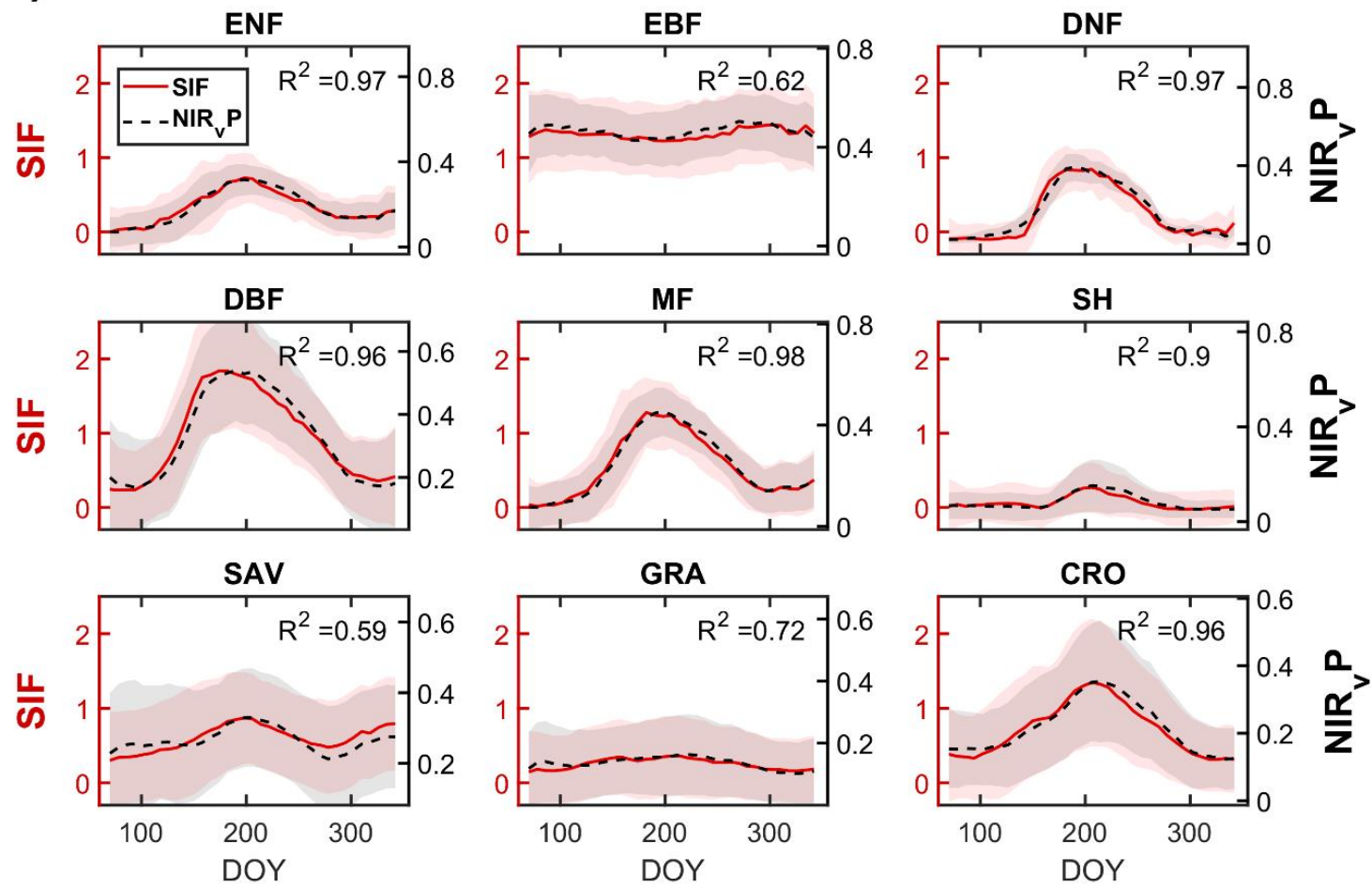

Fig. 3: Global-scale temporal dynamics and correlations of SIF and NIRV. SIF retrievals and NIRV $P$ based on the spaceborne TROPOMI instrument are shown for the year 2018. a) Per-pixel temporal correlation based on 8-day data at 0.05 degree spatial resolution. b) Mean SIF and NIRVP for each plant functional type: evergreen needleleaf forest (ENF), evergreen broadleaf forest (EBF), deciduous needleleaf forest (DNF), deciduous broadleaf forest (DBF), mixed forest (MF), shrubland (SH), savanna (SAV), grassland (GRA) and cropland (CRO). The shading around the mean lines indicates one standard deviation. DOY stands for day of the year. SIF is shown in units of $m W m^{-2} \mathrm{sr}^{-1} \mathrm{~nm}^{-1}$ and NIRVP in units of $\mathrm{nmol} \mathrm{m} \mathrm{m}^{-2} \mathrm{~s}^{-1}$. 
Satellite observations. At the global scale, we found strong temporal correlations between SIF and NIR $P$ (Fig. 3). The temporal SIF-NIR $P$ correlation of 8 -day composite data at 0.05 degree spatial resolution was very strong $\left(R^{2}>0.80\right)$ over large areas, especially in the northern hemisphere and for deciduous forests and crops (Figs. 3a). The temporal regression slopes showed spatial variations (Fig. S3a). When spatially averaging global data for each plant functional type (PFT), SIF and NIRvP showed high temporal correlations, particularly for deciduous forests, evergreen needleleaf forests, crops and shrubland $\left(R^{2} \geq 0.9\right.$, Fig. $\left.3 b\right)$. For evergreen broadleaf forests, the correlation was lower $\left(R^{2}=0.62\right)$, but this was mostly due to the smaller seasonal variations of SIF which were well captured by NIRvP both globally and in the Amazon (Fig. S4a).

Apart from the temporal correlations, we found strong spatial correlations between SIF and NIR $P$ at the global scale $\left(R^{2}=0.78\right.$, Fig. $\left.4 a, c\right)$. In particular, NIR $P$ captured spatial SIF variations very well in North America where SIF shows very high values in the US Corn Belt $\left(R^{2}=0.84\right.$, Fig. 4a,c) and the part of Eurasia that shows a band of high SIF values $\left(R^{2}=0.77\right.$; Fig. $\left.4 b, c\right)$. The spatial and spatio-temporal SIFNIRvP correlations were high throughout the growing season in Europe, the US Corn Belt and globally (mostly $\mathrm{R}^{2}$ around 0.8 at 0.05 degree resolution; Figs. 5, S4), while monthly spatial correlations were somewhat lower for East Asia after the onset of the Monsoon (Fig. S6c, e). The spatial correlation for July and the spatio-temporal correlation of SIF vs. NIRvP over the growing season showed indications of a weak nonlinearity towards high values at the global scale (Figs., 4c, 5c). Spatial regression slopes differed between PFTs and partly also showed considerable seasonal variations (Fig. S5, S6).

NIRvP had stronger spatial and temporal correlations with SIF than other variables that have previously been reported to be good SIF proxies such as APAR ${ }^{14,42}$, EVI2P, and FCVIP 32 (Fig. S7). Both for TROPOMI SIF retrievals and the machine learning product $\mathrm{CSIF}^{43}, \mathrm{NIR}_{\mathrm{V}} \mathrm{P}$ had the highest spatial and temporal correlations to SIF followed by EVI2P, FCVIP, APAR and NDVIP (Fig. S7). The performance rankings were consistent for spatial and temporal correlations, but performance differences between structural SIF proxies were larger for the spatial correlation. APAR and NDVIP had comparable temporal correlations but showed differences for the spatial correlation. 

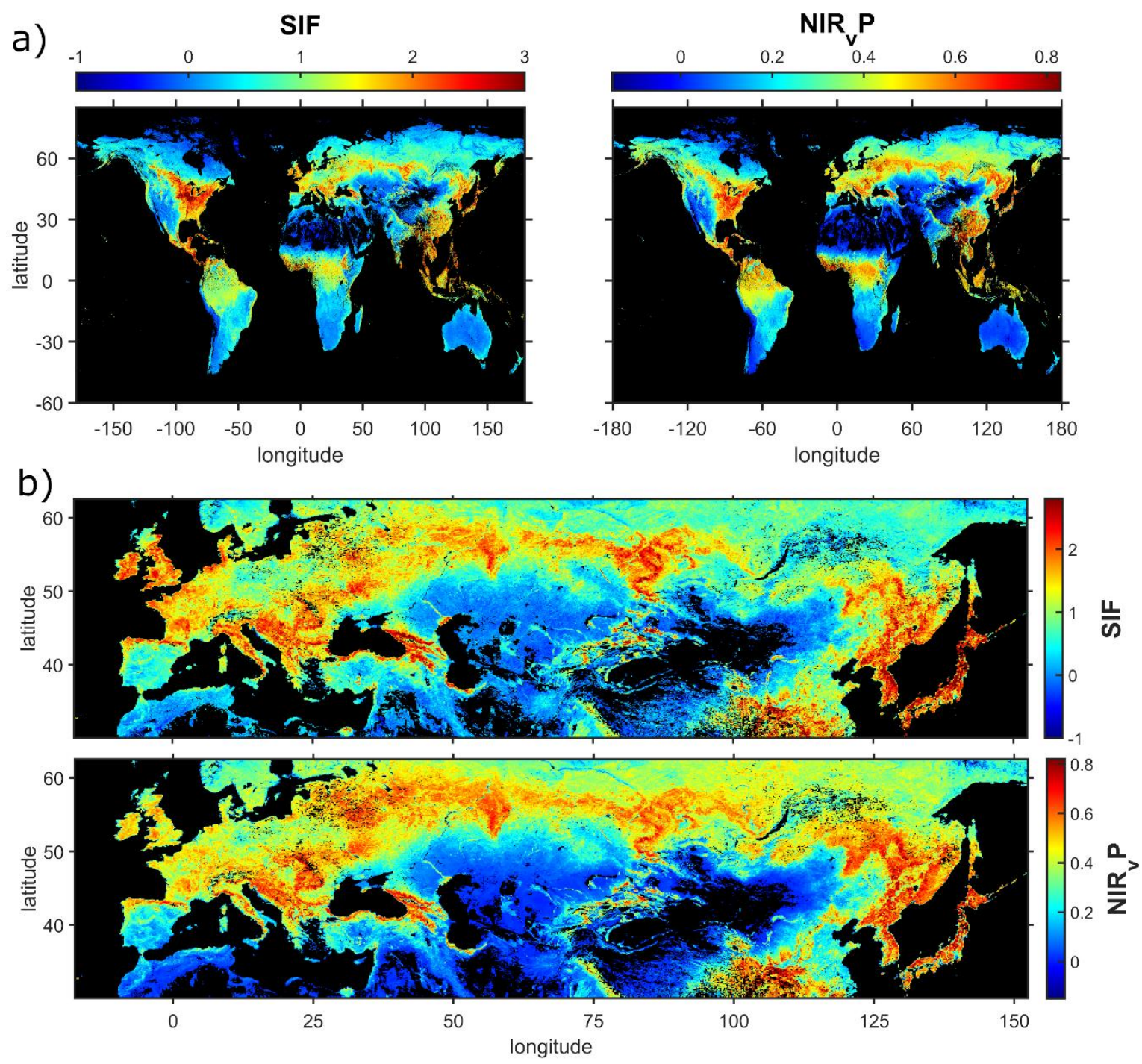

c)
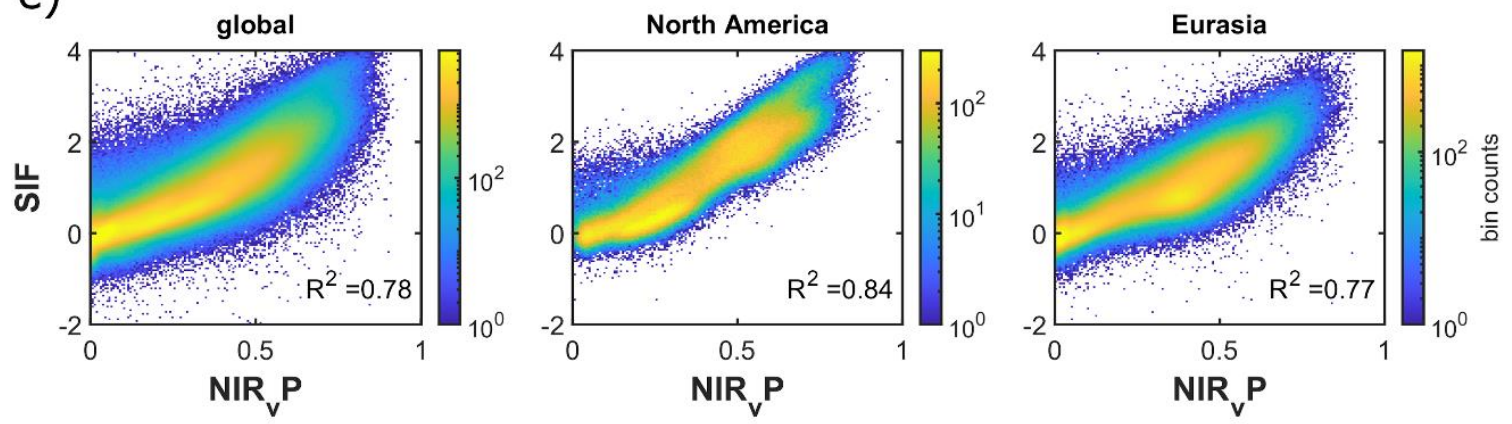

Fig. 4: Global-scale spatial patterns and relationships of SIF and NIRvP in July 2018. Data are from TROPOMI averaged over the month of July at a spatial resolution of 0.05 degree. a) Global maps and b) zoom on part of Eurasia with high SIF values, c) scatter plots of the global and Eurasia panels correspond to the maps shown in a) and c), while the North America panel is based on the geographical selection as in Fig. 5b; the color scale in c) indicates bin counts. SIF is shown in units of $\mathrm{mW} \mathrm{m}^{-2} \mathrm{sr}^{-1} \mathrm{~nm}^{-1} a \mathrm{and} \mathrm{NIRV} \mathrm{P}$ in units of $\mathrm{nmol} \mathrm{m} \mathrm{m}^{-2} \mathrm{~s}^{-1}$. 
a)
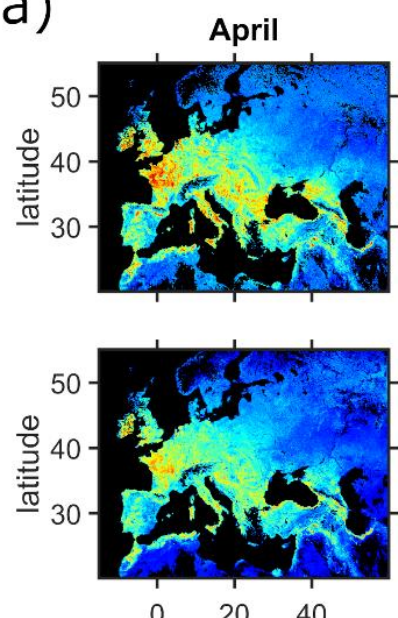

b)
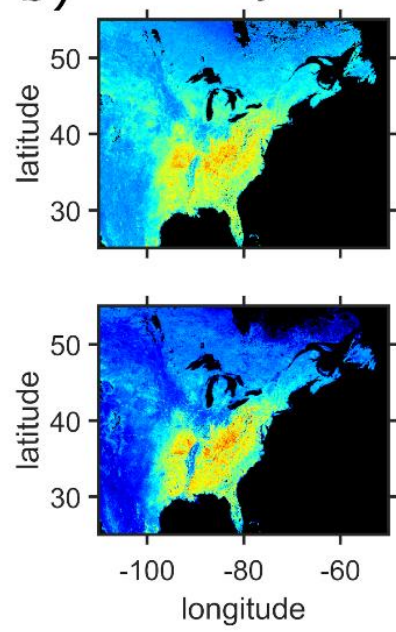

c)

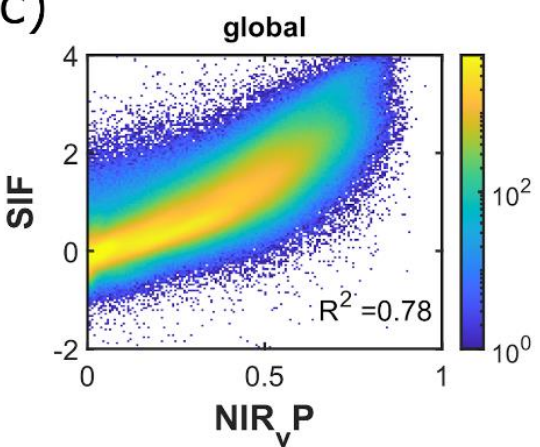

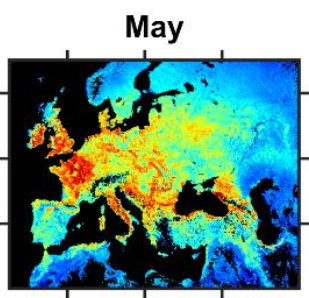
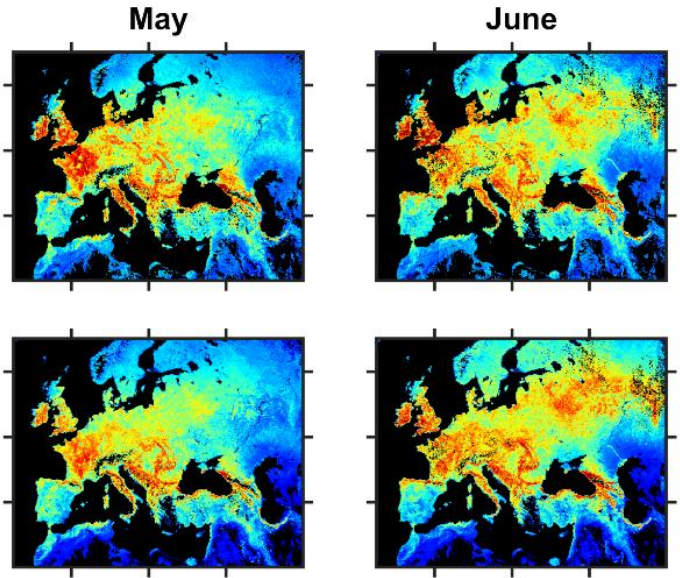

40
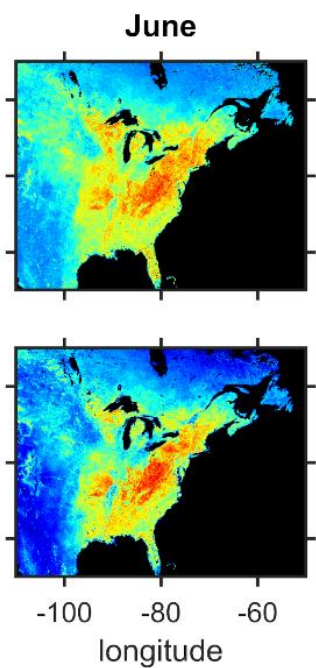

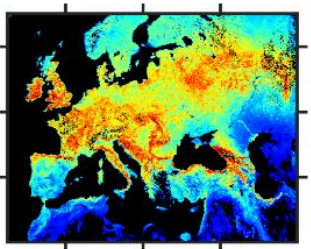

$020 \quad 40$
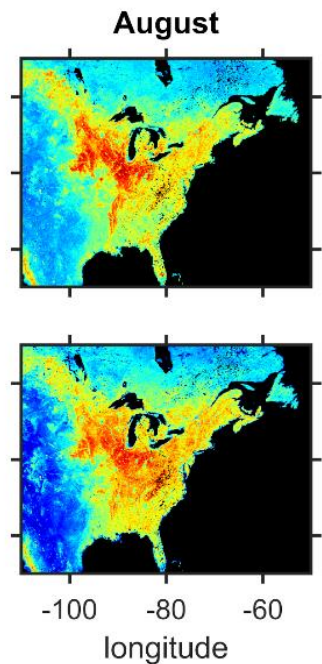
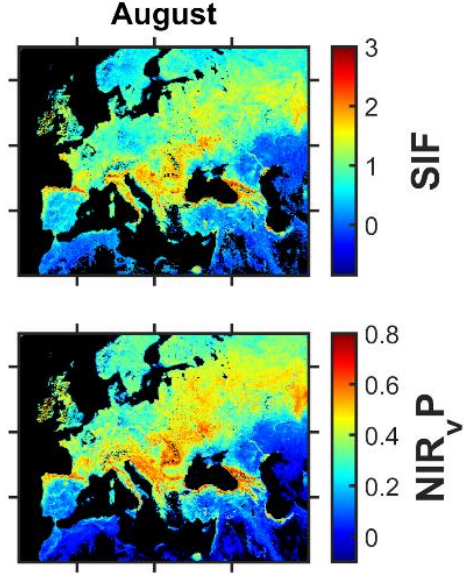

$0 \quad 20 \quad 40$
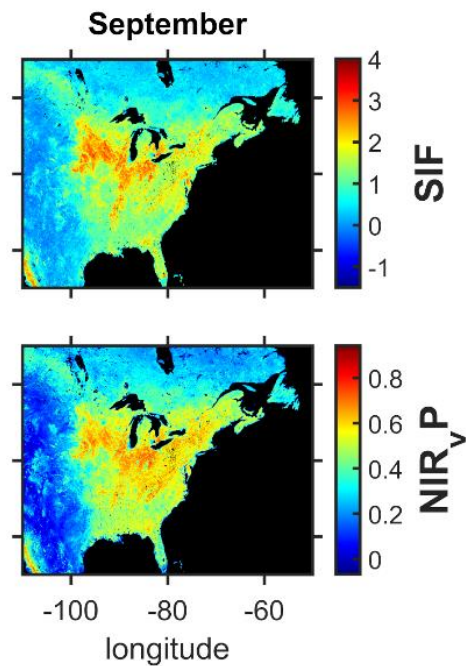

Fig. 5: Spatio-temporal patterns and relationships of SIF and NIRVP in Europe and North America. Monthly average TROPOMI data at 0.05 degree spatial resolution covering the main dynamics of the growing season. a) Europe and the b) North America focusing on the US Corn Belt, c) scatter plots based on monthly data for the main growing season from March to October. All data was evaluated at the spatial resolution of 0.05 degree. SIF is shown in units of $\mathrm{mW} \mathrm{m}^{-2} \mathrm{sr}^{-1} \mathrm{~nm}^{-1}$ and NIRvP in units of nmol m${ }^{-2} \mathrm{~s}$ 
Relationships to GPP. As SIF is commonly used as a proxy for GPP at large scales, we also evaluated the relationships of TROPOMI-based SIF and NIR ${ }_{\mathrm{v}} P$ to FLUXCOM GPP ${ }^{44,45}$ at the global scale (see Methods). We found that NIRvP was highly correlated to GPP and clearly outperformed SIF for both spatial, temporal and spatio-temporal correlations (Fig. 6). Temporal aggregation led to larger increases in the correlation to GPP for SIF than for NIRvP (Fig. 6). This was apparent for both spatial and temporal correlations but was more pronounced in the spatial case. The pattern of increasing correlation between SIF and GPP closely mirrors that of the corresponding SIF vs. NIRvP relationship, especially in the temporal domain (Fig. 6c). The correlation and slopes for GPP relationships across PFTs were very similar for SIF and NIRvP (Figs. S3b,c; S8a) but the variability in slope was considerably larger for SIF than for NIRvP (Fig. S8b). NIRvP showed the strongest correlation to GPP among structural SIF proxies and the patterns of correlation strength to GPP were similar to those for SIF (Fig. S7). 

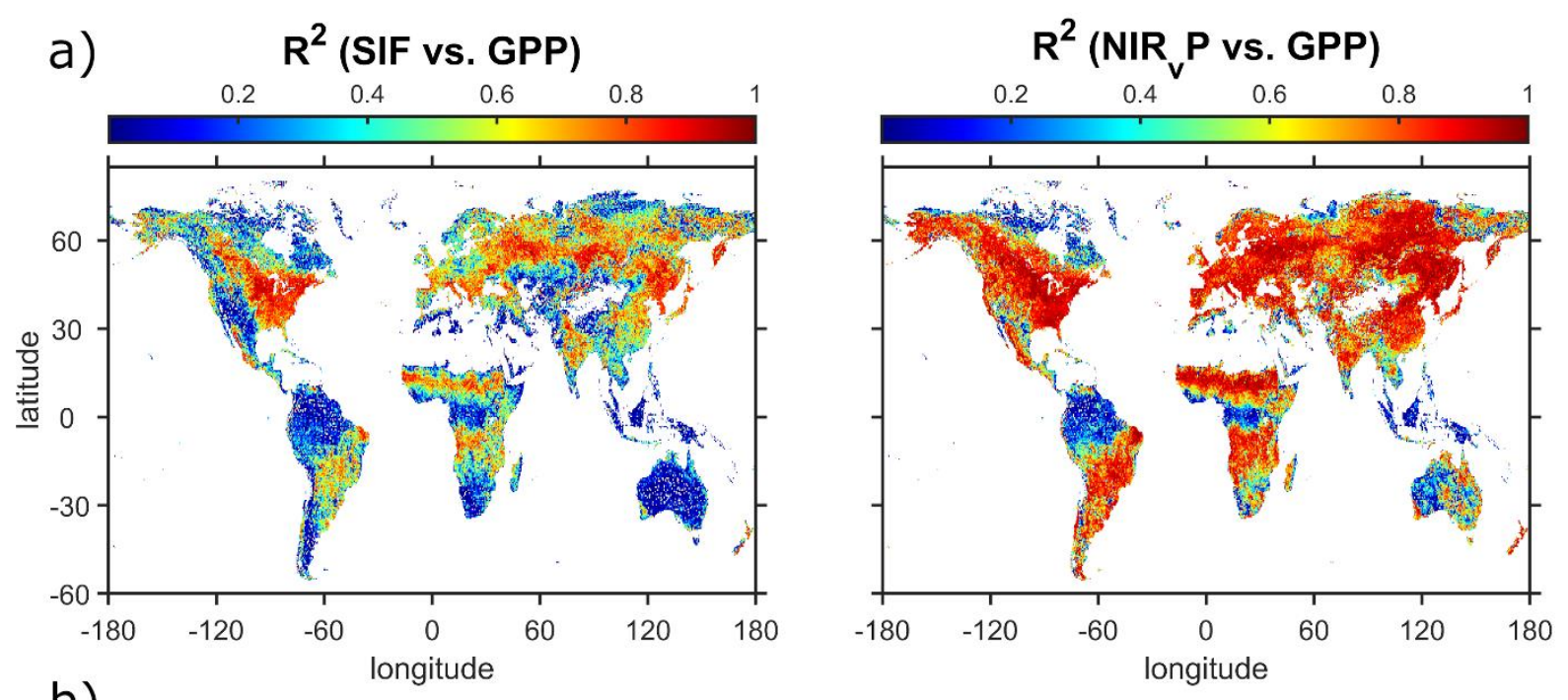

b)
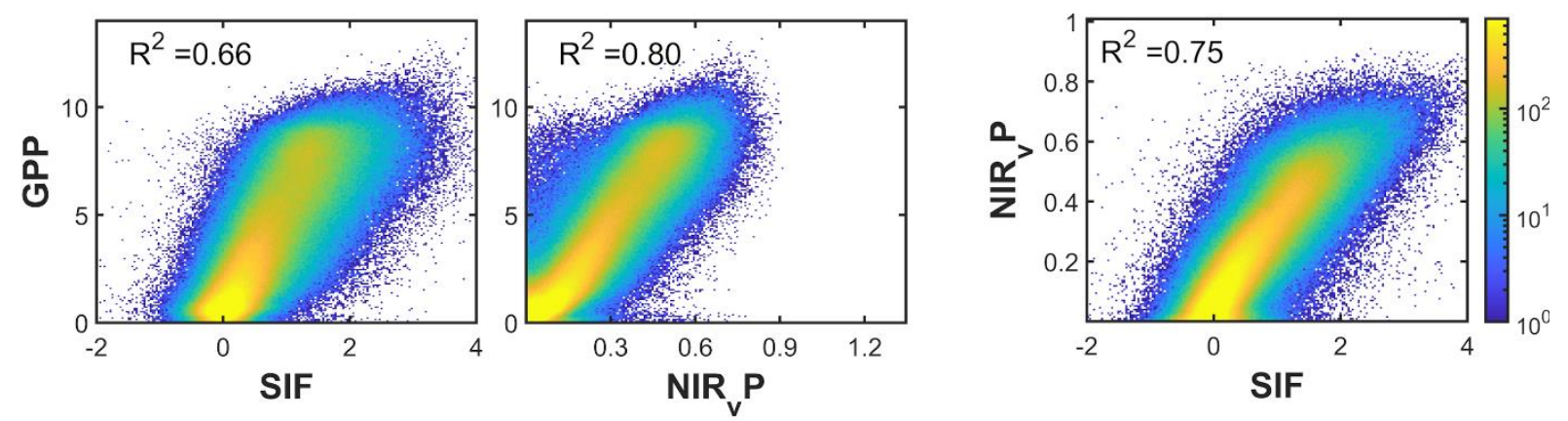

c)

spatial correlation
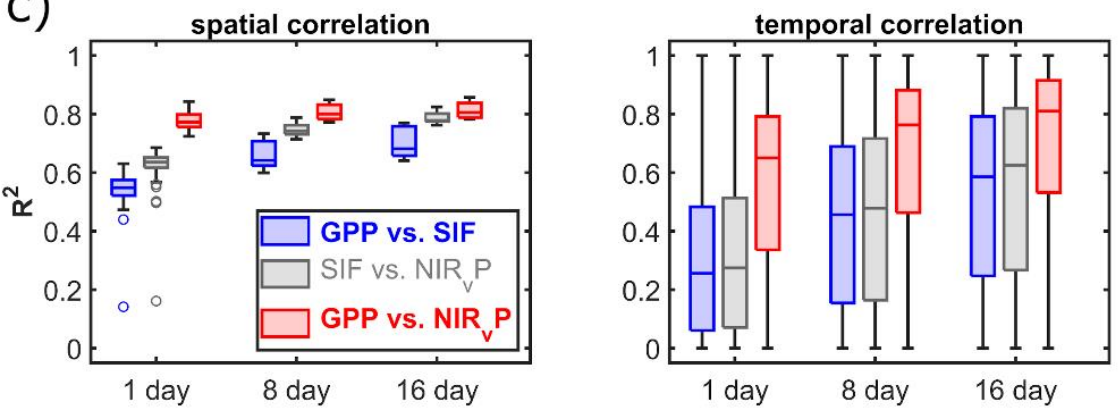

Fig. 6: Global-scale temporal and spatio-temporal correlations between SIF or NIRV to GPP. SIF and NIR $R_{V}$ are based on TROPOMI and for GPP the FLUXCOM RS+METEO ensemble product was used. All results are based on data with 0.5 degree spatial and 8-day temporal resolution. a) temporal correlation maps, b) spatio-temporal correlations between either SIF, NIRVP and GPP for all available data in 2018; color in b) indicates point density (bin count). GPP is given in units of $g \mathrm{C} \mathrm{m}^{-2} \mathrm{~d}^{-1}, \mathrm{SIF}$ in $\mathrm{mW} \mathrm{m}^{-2} \mathrm{sr}-1$ $\mathrm{nm}^{-1}$, and NIRVP in $\mathrm{nmol} \mathrm{m} \mathrm{m}^{-2} \mathrm{~s}^{-1}$. 


\section{Discussion}

Overall, our analysis shows that NIR $\mathrm{P}$ is a robust structural proxy of far-red SIF across ecosystems, spatial and temporal scales and instrument platforms (Figs. 1-5). In particular, the SIF-NIR ${ }_{\vee} P$ relationship holds at very high spatial and temporal resolutions (Figs. 1 and 2), across different crop management regimes (e.g. irrigated vs. rainfed), at a Mediterranean grassland site that experiences very dry conditions (Fig. 1), and for a series of phenotyping plots where physiological variations are expected to be important (Fig. 2 b,c). Our results indicate that the physiological component in SIF, i.e. the $\Phi_{F}$ term, varies little compared to the canopy structure and radiation component of SIF, i.e. APAR $x f_{e s c}$, and that this structural component is well captured by NIRVP (see Methods). More specifically, $\Phi_{\mathrm{F}}$ appears to not only vary considerably less than APAR, which has been previously established ${ }^{14,21}$, but $\Phi_{\mathrm{F}}$ seems to also vary considerably less than $f_{\text {esc }}$ (Figs. $\left.1, S 1\right)^{22,23}$. Therefore, ignoring variations in $\mathrm{fesc}^{46}$ can lead to a misinterpretation of discrepancies between APAR and $\mathrm{SIF}^{22}$, as those discrepancies might be wrongly attributed to $\Phi_{F}$. Results from a process-based model (Fig. S9) further confirmed our observation-based findings and demonstrates that our interpretation of SIF being dominated by canopy structure and radiation is consistent with the current theoretical understanding of $\mathrm{SIF}^{27,28}$ in addition to being supported by empirical evidence from previous studies $22,23,32,33$. The predominance of structure and radiation in explaining spatio-temporal variations of SIF implies that many research applications that use SIF directly, i.e. without extracting or considerably enhancing its small physiological component, could instead use NIRvP without large reductions in performance, and potentially even some improvements ${ }^{12}$.

We found that NIRvP is also a robust structural proxy for GPP and, somewhat unexpectedly, NIRvP outperformed SIF in estimating global GPP at different spatio-temporal scales (Figs. 6, S1d). From a theoretical standpoint, the shared structure and radiation components of SIF and NIRv $P^{19}$, combined with relatively small variations in $\Phi_{\mathrm{F}}{ }^{22}$, mean we should expect similar performance of SIF and NIRVP for GPP estimation supposing ideal, non-noisy signals. However, in contrast to NIRvP, which tends to have high signal quality, SIF is known to be affected by considerable retrieval noise ${ }^{3,4}$ (Table 1). Several aspects of our results support the interpretation that differences in signal quality might explain the weaker GPP estimation performance of SIF compared to NIR P. First, the temporal aggregation results (Fig. 6c) suggest that SIF suffers from considerable noise while NIR、P and FLUXCOM GPP both have much higher signal quality. Noise is reduced with temporal aggregation and correlation strength is expected to considerably increase as a consequence, which matches the observed patterns of correlations involving SIF. For the 
correlation between NIRvP and GPP, however, the increase with temporal aggregation was much less pronounced, especially for the spatial correlation. Second, the close relationship between PFT-level patterns of slopes and correlation in the SIF-GPP and NIRvP-GPP relationships indicate strong similarities between SIF and NIRvP despite the differences in overall correlation (Figs. S3b, S8a). In our findings, only the much larger coefficient of variation in the SIF-GPP slopes compared to the NIRvP-GPP slopes aggregated to PFT level (Fig. S8b) cannot be easily explained by invoking SIF retrieval noise and therefore other potential reasons for these differences should be investigated. Our results on the direct comparison of SIF and NIRvP for GPP estimation confirm and considerably extend previous findings from site-level studies $^{22,40,41}$. Among other things, we confirmed that previous site-level results showing better GPP estimation performance of NIRvP compared to SIF in $\operatorname{crops}^{22,40}$ also held in a Mediterranean grassland site which experiences drought (Fig. S1d). However, since NIRvP is entirely based on structure, it is expected to not fully capture fast physiological responses of photosynthetic light use efficiency to short-term drought and heat stress where canopy structure is relatively stable.

Table 1: Comparison of strengths and weaknesses of SIF and NIR $\boldsymbol{P}$. The comparison addresses important aspects related either to data acquisition, quality and availability, or application for vegetation monitoring. + indicates an advantage, - a disadvantage and the number of + symbols provide a rough indication of the relative advantage over the other variable. 'Ease of measurement' refers to required complexity and performance levels of instruments as well as their cost, 'data availability' focusses on long-term data with high spatio-temporal resolution; 'signal quality' refers to the signal-to-noise-ratio of currently available satellite products; 'background signals' stand for non-vegetated surfaces such as soil, or impervious surfaces in urban areas; 'vegetation structural information' refers to canopy structure characteristics such as leaf area index, clumping and leaf inclination angle, 'vegetation physiological information' refers to signals related to dynamic changes in leaf photosynthetic activity.

\begin{tabular}{lccccccc} 
& $\begin{array}{c}\text { Ease of } \\
\text { measurement }\end{array}$ & $\begin{array}{c}\text { Data } \\
\text { availability }\end{array}$ & $\begin{array}{c}\text { Signal } \\
\text { quality }\end{array}$ & $\begin{array}{c}\text { Insensitivity } \\
\text { to clouds }\end{array}$ & $\begin{array}{c}\text { Insensitivity to } \\
\text { background } \\
\text { signals (soil) }\end{array}$ & $\begin{array}{c}\text { Vegetation } \\
\text { structural } \\
\text { information }\end{array}$ & $\begin{array}{c}\text { Vegetation } \\
\text { physiological } \\
\text { information }\end{array}$ \\
\hline SIF & - & - & - & + & ++ & ++ & ++ \\
NIRvP & ++ & ++ & ++ & - & + & ++ & -
\end{tabular}

Despite the robust SIF-NIRvP correlation across a wide range of spatial and temporal scales, we found differences between the two variables. Conceptually, such differences could be due to differences in data acquisition and processing of SIF and NIRvP, e.g. due to the use of different sensors, limitations in signal quality of either variable, limitations of NIRvP as an approximation of the structure and radiation component of SIF, or the physiological variations in SIF which are not captured by NIR $\mathrm{P}$. We tried to minimize differences in data acquisition and processing by using observations from the same sensors and found that this clearly improved the SIF-NIRvP relationship for airborne and satellite data (Fig. S2b, S7b). 
Several of our results indicate that the lower signal quality of SIF is an important factor explaining observed differences between SIF and NIRVP. In addition to the GPP estimation results discussed above (esp. Fig. 6c), relevant results regarding the retrieval noise in SIF include higher correlations of NIRvP to enhanced machine learning based SIF products compared to original SIF retrievals (Fig. S7b) and extremely strong and linear SIF-NIRvP relationships based on simulated, noise-free data (Fig. S9). Apart from the SIF retrieval noise, we found a strong effect of clouds on the SIF-NIRvP relationship and despite a rather strict filtering (see Methods) cloud contamination still affected the results, especially for East Asia during the monsoon period that starts in July (Fig. S6a) and the tropics (Fig. S5). A stricter filtering would have limited the data availability for our analysis.

Setting aside discrepancies between SIF and NIR $\mathrm{P}$ related to signal quality or the use of different sensors, we found some indications of a slight saturation of NIRvP at high values of SIF for spatial and spatio-temporal relationships (Figs. 2c, 4c, 5c). However, the apparently similar saturation patterns seem to be due to different factors for the airborne and satellite data. For the airborne data, the saturation of NIRvP appears to be inherited from NDVI (Fig. S2c), which might indicate limitations of NIRvP to capture the APAR $x \mathrm{f}_{\mathrm{esc}}$ component of SIF. For the satellite data, in contrast, we found a saturation in spatial and spatio-temporal SIF-NIR $\mathrm{P}$ relationships only for the global scale when combining all data (Figs. 4c, 5c), but not for large regions such as North America, Europe and Eurasia or data grouped by PFT (Figs. 4c, 5c, S7). This suggests that saturation at the global scale results from differences in the SIF-NIRVP slope across regions, e.g. North America vs. Eurasia (Figs. 4c,5c), which, when data are combined can cause apparent saturation. The slope differences between large geographical regions such as North America vs. Europe are likely caused by different fractional coverage in terms of PFTs, as slopes differed between PFTs (Fig. S5) but differences in seasonal slope variation for a given PFT might also contribute. The saturation effects for both the airborne and satellite data were clearly reduced when aggregating to coarser spatial scales (Figs. S2d; Fig. S3 center panel vs. Fig. 5c left hand panel) and the spatio-temporal, global-scale saturation was not consistently apparent in all months (Fig. S6d). It should also be mentioned that the high level of spatial aggregation appears to partly explain the difference between low or moderate pixel-level (Fig. S3b) and very high PFT-level SIF-NIRvP correlations (Fig. 3b), especially for evergreen needleleaf forests where stronger differences are expected based on known physiological mechanisms ${ }^{29,30}$. Therefore, detailed analyses at finer spatial scales are needed. Such studies should consider using enhanced SIF products with higher signal quality and spatio-temporal resolution ${ }^{43,47}$ or higher quality SIF retrievals that will be available in the future from new satellites ${ }^{2,48}$. 
The variation of regression slopes between SIF and NIRVP deserves special consideration as it is theoretically related to variations in $\Phi_{F}$ (see Methods, Eqn. 4). We observed considerable spatial and temporal variations of SIF-NIRvP regression slopes at large scales (Figs. S4, S3, S7). When aggregated to the level of PFTs, the regression slopes partly agree with spatial variations in photosynthetic capacity, $\mathrm{V}_{\mathrm{cmax}}$, especially when using a fixed intercept (Fig. S10). This is consistent with the known sensitivity of SIF and $\Phi_{\mathrm{F}}$ to $\mathrm{V}_{\mathrm{cmax}}{ }^{49-51}$ and indicates the potential usefulness of combining SIF and NIRVP to extract $\Phi_{\mathrm{F}}$. In contrast to the global scale, the variations in regression slopes at the site level (Fig. S1a) could mostly be caused by differences in instruments and SIF retrieval methods (see Table M1 in the Methods section) which are known to considerably affect SIF magnitude ${ }^{31}$. In the spatial domain, similar regression analyses as for the temporal domain could be conducted but we chose to use the simpler approximation by calculating the ratio of SIF/NIRVP instead as regression intercepts tended to vary relatively little and for the airborne data the regression approach is not applicable. At the global scale, we observed considerable temporal variations of the PFT-level SIF/NIRvP ratio (Fig. S4c) that appear to be meaningful in terms of seasonal leaf dynamics of leaf physiology. Interestingly, the temporal variations of SIF/NIRvP seem to peak earlier in the growing season compared to previously reported temporal variations of chlorophyll content $^{52}$ which is also known to covary with photosynthetic capacity ${ }^{53}$. This apparent discrepancy in seasonal dynamics of different remotely sensing-based proxies of $V_{c m a x}$ will require further evaluation, ideally on the basis of ground observations. At the landscape scale, we found considerable differences in the SIF/NIRvP ratio between crop fields based on the high-resolution airborne data (Fig. S2e) that might reflect differences in leaf physiology. Although our results appear promising regarding $\Phi_{\mathrm{F}}$ estimation and its potential for vegetation monitoring, further detailed investigation will be necessary to evaluate the quality of $\Phi_{\mathrm{F}}$ estimates and better understand its variations regarding physiological mechanisms.

We found that, overall, NIRvP showed stronger correlations to SIF compared to the other structural SIF proxies (Figs. S1c, S2a, S7a). In particular, the consistently lower SIF-APAR correlations compared to SIF-NIRvP correlations indicates an important role of $\mathrm{f}_{\text {esc. }}$. At the site level, we used total APAR rather than the so called green APAR, which is the actually relevant part for SIF. However, this can unlikely explain the large differences in seasonal dynamics of APAR compared to SIF (Fig. S1a) as the differences between green and total APAR are expected to be strongest in the senescence phase of $\mathrm{crops}^{54}$. Regarding the better performance of NIRVP compared to FCVIP and EVI2P, we found indications that the latter two are more strongly affected by soil background in very sparse vegetation. This effect could be partly seen from site-level results where the difference to NIRvP was largest for the grassland site (Fig. S1c), which has low 
LAl for a considerable part of the year, but was most apparent at the global scale where soil background characteristics are most variable (Fig. S7c). Despite its better performance compared to other structural SIF proxies, NIRvP is still affected by soil background signals for very sparse vegetation which, together with lower SIF signal quality, could potentially explain lower SIF-NIRvP temporal correlations in shrublands, savanna and grassland ecosystems (Figs. 2a, S3a). Some straightforward practical strategies to further reduce the soil background signal in NIRvP have already been proposed recently ${ }^{19,38}$. However, although simple and robust, the NDVI-based approach to estimating the near-infrared reflectance of vegetation is limited both in terms of the degree of soil background suppression and the selection of the chlorophyllrelated, 'green' vegetation signal. To overcome these limitations, more refined approaches could be developed that might make use of hyperspectral observations to better separate the soil and (green) vegetation signals. NIRv alone, i.e. without taking PAR into account, showed strong performance at the global scale (Fig. S7b), but more detailed analyses revealed that NIRv considerably overestimates SIF in fall when vegetation is still green but PAR decreases (Fig. S4b). These findings indicate a clear advantage of NIRvP over NIRv even at longer time scales (e.g. weekly to monthly) due to seasonal variations of PAR. It should be noted, however, that the better performance of NIRvP compared to NIRv for satellite snapshot observations may not be apparent when comparing data from different satellites due to differences in overpass times and, potentially, observation geometry that affects the atmospheric transmission of upwelling light to the sensor (Fig. S7b). Differences between NIRvP and NIRv regarding their correlation to SIF and GPP are expected to become more important when using data from upcoming geostationary satellite missions such as GeoCarb, TEMPO and Sentinel-4 ${ }^{2}$. As canopy-level PAR cannot be directly observed from airplanes or satellites, either a simple approach that approximates PAR via the downwelling NIR radiance ${ }^{22,37,40}$, or more complex methods involving atmospheric radiative transfer modelling ${ }^{38,55}$ or machine learning ${ }^{56}$ can be used. The radiance-based approach has previously been shown to have comparable performance with direct PAR observations at the site level ${ }^{22}$.

All evidence indicates that SIF and NIRvP are complementary measurements. SIF offers two distinct advantages for improved vegetation monitoring. First, the characteristics of its emission and retrieval make SIF insensitive to soil (emission) and less sensitive to thin clouds (retrieval) than the reflectance or radiance measurements involved in $\mathrm{NIR}_{\mathrm{V}} \mathrm{P}^{57}$ (Table 1). Second, SIF contains unique physiological information in the form of $\Phi_{\mathrm{F}}^{7,29}$. $\Phi_{\mathrm{F}}$ is thought to explain the faster and stronger stress response of SIF compared to structural variables such as APAR, NDVI or EVI (and, in all likelihood, also NIRVP) as observed in extreme events such as drought or heat waves ${ }^{21,26,35,36,58}$. However, these apparent advantages of SIF 
can be offset by considerable practical limitations in terms of data availability, spatio-temporal resolution and signal quality ${ }^{1,12,59}$ (Fig. 6). NIR P, in contrast, has long term data records ${ }^{39}$ with high signal quality and, increasingly, very high spatio-temporal resolution ${ }^{33,38}$ (Table 1) and therefore has advantages over SIF with respect to its structure and radiation component. Apart from the individual advantages of SIF and NIRvP, they can be effectively used in combination for at least two purposes. First, the ratio SIF/NIRvP or the corresponding regression slope can be used to estimate the physiological component of SIF, $\Phi_{\mathrm{F}}{ }^{22}$ (see Methods and Figs. S3d, S10). As $\Phi_{\mathrm{F}}$ estimation amplifies SIF retrieval noise ${ }^{22}$, however, very high quality SIF products should be used to avoid the need for aggregation to coarser scales. Second, since SIF and NIR $\mathrm{V} P$ share the same structure and radiation components (APAR $\times f_{e s c}$ ) and NIR $P$ typically has higher signal quality than SIF, evaluating the SIF-NIR $P$ relationship can be used to assess the quality of SIF retrievals beyond diurnal variations that are strongly driven by $P A R^{31}$. NIRvP might therefore prove helpful in further improving SIF retrieval methods as they continue to be refined ${ }^{31,60,61}$.

Overall, our study demonstrates the importance of canopy structure and solar radiation for understanding variations of SIF and GPP over a large range of spatio-temporal scales. We therefore expect NIRvP, which can capture most of these variations, to be more widely applied in future research on remote estimation of GPP, crop yield modelling and other related subjects. Furthermore, making more effective use of the physiological information in SIF by extracting it with the help of NIR $\mathrm{P}$ might result in improved GPP estimation and new insights on vegetation dynamics. 


\section{Materials and Methods}

\section{Theoretical framework}

Canopy-level far-red SIF, which we exclusively consider in this manuscript, can be decomposed into three mechanistic components, namely the absorbed fraction of photosynthetically active radiation (APAR), the canopy escape fraction, and the fluorescence emission yield, $\Phi_{\mathrm{F}}{ }^{46}$ :

$$
S I F_{o b s}=A P A R \times f_{e s c} \times \Phi_{F}
$$

Our study relies on the previously established result ${ }^{19}$ that, except for very low fractional vegetation cover, $f_{\text {esc }}$ for far-red SIF, which we exclusively consider in this manuscript, can be well approximated in the following way:

$$
f_{e s c} \approx \frac{N I R_{V}}{f P A R}
$$

where $P P A R$ is the fraction of photosynthetically active radiation absorbed and NIRv is the near-infrared reflectance of vegetation estimated as NDVI $\times \mathrm{NIR}^{33}$, where NIR stands for near-infrared reflectance and NDVI is the Normalized Difference Vegetation Index ${ }^{62,63}$. When substituting Eqn. 2 into Eqn. 1, using APAR $=$ FPAR $\times$ PAR, and using the definition

$$
N I R_{V} P=N I R_{V} \times P A R
$$

we obtain the approximation

$$
S I F_{o b s} \approx N I R_{V} P \times \Phi_{F}
$$

which is the basis of our rationale that SIF can be approximated by NIR $\mathrm{P}$ assuming relatively small variability of $\Phi_{F}$ compared to the variability of $N I R_{V} P$. It is clear from Eqn. 4 that $\Phi_{F}$ can be estimated as the ratio of SIF/NIR $\mathrm{R}_{\mathrm{V}} \mathrm{P}$ or as the slope in the linear regression of SIF vs. NIRVP. Including an intercept term in the regression can account for either imperfect SIF retrieval (i.e. an offset), soil background impacts on NIRvP, or both.

We use the convenient shorthand notation introduced in Eqn. 3 also more generally for other vegetation indices (VI) that we consider potential structural SIF proxies in the way VIP = VI $\times$ PAR. Thus, NDVI becomes NDVIP etc. which is also used for $\mathrm{EVI}^{64}$ and $\mathrm{FCVI}^{32}$. 


\section{Data sets and processing}

Site data. We used previously published data from a total of six sites located in South Korea (rice) ${ }^{14}$, China (corn 1) ${ }^{65}$, France (wheat) ${ }^{66}$, Spain (natural grassland) ${ }^{16,67,68}$ and the United States (corn 2 and soybean) ${ }^{40}$. Only the rice paddy, corn 2 and soybean sites were irrigated/flooded. Tower-mounted spectrometers with sub-nanometer spectral resolution were used for SIF retrieval at all sites. Except for the wheat site that used the TriFLEX instrument ${ }^{69}$ and the grassland site which used a commercial FLoX instrument (JB Hyperspectral Devices, Düsseldorf, Germany) all other sites had set-ups similar to FluoSpec2 ${ }^{70}$ based on the QEPro spectroradiometer (Ocean Optics, Dunedin, FL, USA), although with partly different spectral resolutions. An overview of all site-level and larger scale SIF datasets including information on the retrieval algorithm used is given in Table M1 below. $\mathrm{NIRv}^{33}$ was calculated from co-located spectrometers covering the visible-nearinfrared spectral range at a lower spectral resolution as we did not observe disadvantages compared to using the same sensor as for SIF retrieval and the latter also partly did not cover the red band. NIR and red reflectance bands were calculated as averages of $600-650 \mathrm{~nm}$ and $800-850 \mathrm{~nm}$, respectively, and used to calculate $\mathrm{NIRv}, \mathrm{EVI}^{64}$ and approximate $\mathrm{FCVI} \mathrm{I}^{32}$ as the simple difference vegetation index NIR - red. Except for the rice paddy site which had a hemispheric viewing geometry for the upwelling radiation measurements, all other sites had a narrow angle field of view at nadir. PAR data was acquired with quantum sensors. NIRvP was calculated as NIRv $\times$ PAR. APAR was measured with either quantum (wheat, corn, soy) or LED (rice) sensors above and below the canopy at all sites except the grassland site. For the rice site, fPAR had to be gap-filled for part of the green-up part of the growing season using a radiative transfer model ${ }^{14,71}$. A Hampel outlier filtering (window length of 12 days, threshold parameter equals 3) was conducted for the grassland data to filter out strong outliers in the SIF time series. For all sites, data between 8 am and $4 \mathrm{pm}$ local time were selected. More details on methods and instrumentation can be found in the references given in Table M1.

To investigate if part of our results on the strong SIF-NIRVP relationship are consistent with the current theoretical understanding of SIF, we used simulations with the process-based model SCOPE ${ }^{72,73}$ for the rice paddy site. To obtain a realistic scenario, the simulations were based on in-situ observations of meteorological conditions and relevant vegetation parameters in the rice paddy site. More details can be found in a previous publication from which the simulation outputs were reused here ${ }^{17}$. 
Table M2: Overview of all SIF datasets used in this study. The location is given in units of degrees north (latitude) and east (longitude). The retrieval methods used include Singular Vector Decomposition (SVD), Spectral Fitting Method (SFM), improved Fraunhofer Line Depth (iFLD), and another version of FLD where ' $n$ ' indicates the number of channels used (nFLD).

\begin{tabular}{|c|c|c|c|c|c|c|c|}
\hline $\begin{array}{c}\text { Scale/ } \\
\text { platform }\end{array}$ & $\begin{array}{l}\text { Vegetation } \\
\text { type }\end{array}$ & Instrument & $\begin{array}{l}\text { Retrieval } \\
\text { method }\end{array}$ & $\begin{array}{c}\text { Obs. } \\
\text { geometry }\end{array}$ & $\begin{array}{l}\text { Location } \\
\text { (Lat./Long) }\end{array}$ & year & Literature reference \\
\hline \multirow{6}{*}{$\begin{array}{l}\text { Site/ } \\
\text { tower }\end{array}$} & rice & Fluospec2 & SVD & $\begin{array}{l}\text { Hemi- } \\
\text { spheric }\end{array}$ & $\begin{array}{l}38.2013 / \\
127.2506\end{array}$ & 2016 & K. Yang et al. (2018) \\
\hline & wheat & TriFLEX & $\mathrm{nFLD}$ & Nadir & $\begin{array}{l}43.9175 / \\
4.8797\end{array}$ & 2010 & Goulas et al. (2017) \\
\hline & corn (1) & Fluospec2 & SFM & Nadir & $\begin{array}{l}34.5199 / \\
115.5916\end{array}$ & 2017 & Li et al. (2020) \\
\hline & $\operatorname{corn}(2)$ & Fluospec2 & iFLD & Nadir & $\begin{array}{l}41.1649 / \\
-96.4701\end{array}$ & 2017 & Wu et al. (2019) \\
\hline & soybean & Fluospec2 & iFLD & Nadir & $\begin{array}{l}41.1649 / \\
-96.4701\end{array}$ & 2018 & Wu et al. (2019) \\
\hline & grass & FLoX & SFM & Nadir & $\begin{array}{c}39.9403 / \\
-5.7639\end{array}$ & 2017 & $\begin{array}{c}\text { Migliavacca et al. (2017), } \\
\text { Zhang et al. (2020) }\end{array}$ \\
\hline $\begin{array}{l}\text { Landscape/ } \\
\text { Airborne }\end{array}$ & crops & HyPlant & SFM & Nadir & $\begin{array}{c}50.6167 / \\
6.9833\end{array}$ & 2018 & $\begin{array}{l}\text { Siegmann et al. (2019), } \\
\text { Siegmann (2019) }\end{array}$ \\
\hline $\begin{array}{l}\text { Global/ } \\
\text { satellite }\end{array}$ & All & TROPOMI & SVD & variable & All & 2018 & Köhler et al. (2018) \\
\hline
\end{tabular}

Airborne SIF data. We used data from the high performance airborne imaging spectrometer HyPlant FLUO, which was specifically designed to be used for SIF retrieval ${ }^{74,75}$ and has been used in the preparation for the upcoming FLEX satellite mission ${ }^{48}$. The 2018 dataset we used (Table M1) is based on an improved processing chain, which results in high quality SIF retrievals ${ }^{60,75}$. The data was acquired on June 29, 2018 at 12:30 MEST at $680 \mathrm{~m}$ above ground level in at the agricultural research station Campus Klein-Altendorf in western Germany $\left(50^{\circ} 37^{\prime} \mathrm{N}, 6^{\circ} 59^{\prime} \mathrm{E}\right)$. More details can be found in the relevant references ${ }^{60,75}$. We exclusively used original $1 \mathrm{~m}$ spatial resolution data in all analyses except Fig. S2d. NIRvP was estimated from FLUO at-sensor radiance data to ensure minimal differences compared to SIF retrievals in terms of sensor and processing aspects. The approach of using NIR radiance as proxy for the product of NIR reflectance times PAR was previously introduced as $N I R_{v} R^{22}$ and was found to show good performance in terms of correlation to SIF and GPP at the site level22,40,41. Those results included observations in cloudy conditions where the largest differences between PAR and NIR radiance are expected, the discrepancies between the two variables should be even smaller in the clear sky conditions under which the airborne campaign was conducted. To ensure the smallest impact of the atmospheric path between the canopy and the sensor on our results, we chose the wavelengths for NIR as 756-758 nm, inside an atmospheric window $^{76,77}$. Although the NIR radiance at $756-758 \mathrm{~nm}$ also includes SIF, SIF contributes only several 
percent $^{3}$ to the upwelling NIR radiance and therefore can be neglected for our purpose. Red wavelengths were chosen in the range $670-684 \mathrm{~nm}$ in a compromise to average over a sufficiently large number of bands to increase signal quality but attempting to avoid atmospheric absorption features, especially the oxygen-B band, and shorter wavelengths which are more strongly affected by atmospheric scattering. As no surface reflectance product was available for the FLUO data, we relied on the very strong linear relationships $\left(R^{2}>0.99\right)$ between DUAL at-sensor radiance and DUAL surface reflectance at the relevant wavelengths to convert FLUO at-sensor radiance data to FLUO surface reflectance for the calculation of NDVI, an estimate of FCVI via the difference vegetation index and EVI2. To do so, the linear regression coefficients obtained from the DUAL data were applied to the FLUO observations. To convert units of $\mathrm{mW}$ $\mathrm{m}^{-2} \mathrm{sr}^{-1} \mathrm{~nm}^{-1}$ inherited from the radiance data to units of $\mathrm{nmol} \mathrm{m} \mathrm{m}^{-2} \mathrm{~s}^{-1}$, we used the very strong linear regression relationship $\left(R^{2}=0.98\right)$ between $N I R_{v} P$ and NIRv $R$ at the site level.

Satellite SIF data. We primarily used satellite SIF retrievals from the TROPOMI instrument on Sentinel$5 \mathrm{P}^{76}$. The instantaneous data in 2018 was gridded at 0.05 degree spatial resolution. In an approach that is conceptually similar to the one used for the airborne data (i.e. $N I R_{v} R^{22}$ ), NIRvP was estimated by multiplying the TROPOMI NIR radiance at $759 \mathrm{~nm}$ with MODIS NDVI, which was aggregated to 0.05 degree resolution. MODIS rather than TROPOMI NDVI was used as no surface reflectance product is currently available for TROPOMI and the red band would have to be atmospherically corrected. We used the TROPOMI NIR radiance at $759 \mathrm{~nm}$, as it was provided in the original SIF data product and no atmospheric correction is necessary due to its location in an atmospheric window ${ }^{76,77}$. This latter aspect ensures a direct comparability with the corresponding SIF retrievals as atmospheric correction for NIR at other wavelengths could introduce biases or artefacts leading to discrepancies between SIF and NIRvP. As NIR radiance is very sensitive to clouds, we applied a cloud filtering. For this, we used the VIIRS-based cloud product ${ }^{78}$ with a threshold of 0.35 for the cloud fraction, which can reduce both direct cloud effects and indirect effects on the validity of using downwelling NIR radiance as a proxy for PAR. In addition to the cloud filtering, data with SIF signal uncertainty (1- $\sigma$ retrieval error) larger than $0.55 \mathrm{~mW} \mathrm{~m}^{-2} \mathrm{sr}^{-1} \mathrm{~nm}^{-1}$ as well as SIF values $>4$ or $<-2 \mathrm{~mW} \mathrm{~m}^{-2} \mathrm{sr}^{-1} \mathrm{~nm}^{-1}$ were excluded from the analysis. MODIS NDVI was based on daily red and NIR nadir-adjusted surface reflectance products (MCD43D62, MCD43D63). SIF and NIRvP relationships were evaluated for instantaneous data, 8-day, 16-day and monthly composites. For evaluating results per PFT, we used the MODIS MCD12C1 land cover product. For the comparison of different variables to NIR $\mathrm{P}$ in terms of correlation to SIF, we relied on the same MODIS products as used for NDVI and multiplied the vegetation indices by BESS PAR ${ }^{55}$, which is also based on MODIS products. We 
approximated $\mathrm{FCVI}^{32}$ by using the red band rather than the average of visible reflectance which is not available from MODIS. EVI2 was chosen rather than EVI as the blue band is sensitive to atmospheric correction errors and relying only on red and NIR bands permits a more direct comparison among the three indices (NIRv, FCVI and EVI2) in terms of the equation used. Previous satellite-based results showing better performance of $\mathrm{NIR}_{\mathrm{V}}$ compared to $\mathrm{EVI}^{79}$ suggest that EVIP would not outperform NIRvP. For NIRvP, units of radiance were converted to PAR units in the same way as for the airborne data (see above).

To evaluate the effect of PAR on the SIF-NIRvP relationship, we compared NIRvP to NIRv. Since no surface reflectance product is available for TROPOMI, we normalized at sensor NIR radiance by the cosine of the solar zenith angle following previous studies ${ }^{80}$.

As additional test, we compared MODIS NIRv P with $\mathrm{CSIF}^{43}$, which is a machine learning product based on OCO-2 SIF retrievals, MODIS reflectance and FPAR products. Since the original version of the product did not cover 2018, we used the recently updated version 2 of the product. The CSIF data was available at 0.05 degree spatial and 4-day temporal resolution.

For all satellite analyses, the fixed value 0.1 was substracted from NDVI to partially account for soil background ${ }^{33}$. Negative values of NIRvP and NIRv were excluded as they are typically caused by negative NDVI values related to snow.

Global GPP. To evaluate the SIF-GPP relationship at the global scale, we used the ensemble RS+Meteo FLUXCOM GPP product ${ }^{44,45}$, which uses machine learning algorithms to up-scale eddy covariance tower observations to the globe.

Analyses. For the slope analyses in SIF-NIRVP, and SIF-GPP as well as NIRVP-GPP relationships, we conducted linear regression with either variable or constant intercept as there were artefacts for evergreen broadleaf forest due to the distribution of the data (only high values). The constant intercept was determined by averaging the median intercept in each PFT. For the case of temporal regression, evergreen broadleaf forest was excluded from the intercept calculation.

We relied on squared Pearson correlation as the main performance metric as it is equivalent to the coefficient of determination of linear regression with an intercept and a single explanatory variable. 


\section{Data and code availability}

Site data is available from the respective site PIs upon request. The airborne HyPlant dataset is available for download (https://doi.ipk-gatersleben.de/DOI/3dede5ba-d57a-4cf5-9d06-6fd9a70f52c9/d5a1e61eb0c7-447b-81f5-d94ffad7dc0c/2). TROPOMI SIF retrieval products are also available for download (ftp://fluo.gps.caltech.edu/data/tropomi/). MODIS data can be downloaded from NASA. CSIF is also available for download (https://figshare.com/articles/CSIF/6387494). Custom code for data processing and analysis is available from the authors upon request.

\section{Acknowledgements}

This study was funded by National Research Foundation of Korea (NRF-2019R1A2C2084626). We thank a large number of colleagues for contributing to the site datasets as well as the airborne and satellite SIF products. We are also grateful for the contributions of Chongya Jiang to the BESS modelling platform and thank Yulin Yan, Seungjoon Lee, Bolun Li, and Juwon Kong for downloading and processing MODIS data. We thank Bastian Siegmann for providing useful information and additional data related to the HyPlant dataset. Furthermore, we thank Martin Jung and Ulrich Weber for sharing the FLUXCOM products and Yao Zhang and Pierre Gentine for making the CSIF dataset publicly available.

\section{Author Contributions}

B.D. and Y.R. designed this study. B.D. conducted the analysis with support and feedback from other coauthors. B.D., Y.R. and G.B. wrote the paper with contributions from all other co-authors. 


\section{References}

1. Ryu, Y., Berry, J. A. \& Baldocchi, D. D. What is global photosynthesis? History, uncertainties and opportunities. Remote Sens. Environ. 223, 95-114 (2019).

2. Mohammed, G. H. et al. Remote sensing of solar-induced chlorophyll fluorescence (SIF) in vegetation: 50 years of progress. Remote Sens. Environ. 231, 111177 (2019).

3. Frankenberg, C. \& Berry, J. Solar Induced Chlorophyll Fluorescence: Origins, Relation to Photosynthesis and Retrieval. in Comprehensive Remote Sensing 143-162 (Elsevier, 2018). doi:10.1016/B978-0-12-409548-9.10632-3.

4. Köhler, P., Guanter, L. \& Joiner, J. A linear method for the retrieval of sun-induced chlorophyll fluorescence from GOME-2 and SCIAMACHY data. Atmospheric Meas. Tech. 8, 2589-2608 (2015).

5. Joiner, J. et al. Systematic Orbital Geometry-Dependent Variations in Satellite Solar-Induced Fluorescence (SIF) Retrievals. Remote Sens. 12, 2346 (2020).

6. Gamon, J. A., Penuelas, J. \& Field, C. B. A narrow-waveband spectral index that tracks diurnal changes in photosynthetic efficiency. Remote Sens. Environ. 41, 35-44 (1992).

7. Porcar-Castell, A. et al. Linking chlorophyll a fluorescence to photosynthesis for remote sensing applications: mechanisms and challenges. J. Exp. Bot. 65, 4065-4095 (2014).

8. Walther, S. et al. Satellite chlorophyll fluorescence measurements reveal large-scale decoupling of photosynthesis and greenness dynamics in boreal evergreen forests. Glob. Change Biol. n/a-n/a (2015) doi:10.1111/gcb.13200.

9. Guan, K. et al. Photosynthetic seasonality of global tropical forests constrained by hydroclimate. Nat. Geosci. 8, 284-289 (2015).

10. Qiu, B. et al. Satellite Chlorophyll Fluorescence and Soil Moisture Observations Lead to Advances in the Predictive Understanding of Global Terrestrial Coupled Carbon-Water Cycles. Glob. Biogeochem. Cycles 32, 360-375 (2018). 
11. Guan, K. et al. Improving the monitoring of crop productivity using spaceborne solar-induced fluorescence. Glob. Change Biol. 22, 716-726 (2016).

12. Peng, B. et al. Assessing the benefit of satellite-based Solar-Induced Chlorophyll Fluorescence in crop yield prediction. Int. J. Appl. Earth Obs. Geoinformation 90, 102126 (2020).

13. He, L. et al. From the Ground to Space: Using Solar-Induced Chlorophyll Fluorescence to Estimate Crop Productivity. Geophys. Res. Lett. 47, (2020).

14. Yang, K. et al. Sun-induced chlorophyll fluorescence is more strongly related to absorbed light than to photosynthesis at half-hourly resolution in a rice paddy. Remote Sens. Environ. 216, 658-673 (2018).

15. Yang, X. et al. Solar-induced chlorophyll fluorescence that correlates with canopy photosynthesis on diurnal and seasonal scales in a temperate deciduous forest: Fluorescence and photosynthesis. Geophys. Res. Lett. 42, 2977-2987 (2015).

16. Migliavacca, M. et al. Plant functional traits and canopy structure control the relationship between photosynthetic $\mathrm{CO}_{2}$ uptake and far-red sun-induced fluorescence in a Mediterranean grassland under different nutrient availability. New Phytol. (2017) doi:10.1111/nph.14437.

17. Dechant, B., Ryu, Y. \& Kang, M. Making full use of hyperspectral data for gross primary productivity estimation with multivariate regression: Mechanistic insights from observations and process-based simulations. Remote Sens. Environ. 234, 111435 (2019).

18. Qiu, B., Chen, J. M., Ju, W., Zhang, Q. \& Zhang, Y. Simulating emission and scattering of solarinduced chlorophyll fluorescence at far-red band in global vegetation with different canopy structures. Remote Sens. Environ. 233, 111373 (2019).

19. Zeng, Y. et al. A practical approach for estimating the escape ratio of near-infrared solar-induced chlorophyll fluorescence. Remote Sens. Environ. 232, 111209 (2019). 
20. Miao, G. et al. Sun-Induced Chlorophyll Fluorescence, Photosynthesis, and Light Use Efficiency of a Soybean Field from Seasonally Continuous Measurements. J. Geophys. Res. Biogeosciences 123, 610-623 (2018).

21. Wieneke, S. et al. Linking photosynthesis and sun-induced fluorescence at sub-daily to seasonal scales. Remote Sens. Environ. 219, 247-258 (2018).

22. Dechant, B. et al. Canopy structure explains the relationship between photosynthesis and suninduced chlorophyll fluorescence in crops. Remote Sens. Environ. 241, 111733 (2020).

23. Wang, C. et al. Satellite footprint data from OCO-2 and TROPOMI reveal significant spatio-temporal and inter-vegetation type variabilities of solar-induced fluorescence yield in the U.S. Midwest. Remote Sens. Environ. 241, 111728 (2020).

24. Yang, P. \& van der Tol, C. Linking canopy scattering of far-red sun-induced chlorophyll fluorescence with reflectance. Remote Sens. Environ. 209, 456-467 (2018).

25. Flexas, J. et al. Steady-state chlorophyll fluorescence (Fs) measurements as a tool to follow variations of net $\mathrm{CO} 2$ assimilation and stomatal conductance during water-stress in C3 plants. Physiol. Plant. 114, 231-240 (2002).

26. Jonard, F. et al. Value of sun-induced chlorophyll fluorescence for quantifying hydrological states and fluxes: Current status and challenges. Agric. For. Meteorol. 291, 108088 (2020).

27. Gu, L., Han, J., Wood, J. D., Chang, C. Y. \& Sun, Y. Sun-induced Chl fluorescence and its importance for biophysical modeling of photosynthesis based on light reactions. New Phytol. (2019) doi:10.1111/nph.15796.

28. van der Tol, C., Berry, J. A., Campbell, P. K. E. \& Rascher, U. Models of fluorescence and photosynthesis for interpreting measurements of solar-induced chlorophyll fluorescence. J. Geophys. Res. Biogeosciences 119, 2312-2327 (2014). 
29. Magney, T. S. et al. Mechanistic evidence for tracking the seasonality of photosynthesis with solarinduced fluorescence. Proc. Natl. Acad. Sci. 201900278 (2019) doi:10.1073/pnas.1900278116.

30. Porcar-Castell, A. A high-resolution portrait of the annual dynamics of photochemical and nonphotochemical quenching in needles of Pinus sylvestris. Physiol. Plant. 143, 139-153 (2011).

31. Chang, C. Y. et al. Systematic assessment of retrieval methods for canopy far-red solar-induced chlorophyll fluorescence (SIF) using high-frequency automated field spectroscopy. J. Geophys. Res. Biogeosciences (2020) doi:10.1029/2019JG005533.

32. Yang, P., van der Tol, C., Campbell, P. K. E. \& Middleton, E. M. Fluorescence Correction Vegetation Index (FCVI): A physically based reflectance index to separate physiological and non-physiological information in far-red sun-induced chlorophyll fluorescence. Remote Sens. Environ. 240, 111676 (2020).

33. Badgley, G., Field, C. B. \& Berry, J. A. Canopy near-infrared reflectance and terrestrial photosynthesis. Sci. Adv. 3, e1602244 (2017).

34. Huete, A. et al. Overview of the radiometric and biophysical performance of the MODIS vegetation indices. Remote Sens. Environ. 83, 195-213 (2002).

35. Qiu, B., Ge, J., Guo, W., Pitman, A. J. \& Mu, M. Responses of Australian dryland vegetation to the 2019 heatwave at a sub-daily scale. Geophys. Res. Lett. (2020) doi:10.1029/2019GL086569.

36. Li, X. \& Xiao, J. Global climatic controls on interannual variability of ecosystem productivity: Similarities and differences inferred from solar-induced chlorophyll fluorescence and enhanced vegetation index. Agric. For. Meteorol. 288-289, 108018 (2020).

37. Baldocchi, D. D. et al. Outgoing Near Infrared Radiation from Vegetation Scales with Canopy Photosynthesis Across a Spectrum of Function, Structure, Physiological Capacity and Weather. J. Geophys. Res. Biogeosciences (2020) doi:10.1029/2019JG005534. 
38. Jiang, C., Guan, K., Wu, G., Peng, B. \& Wang, S. A daily, 250 m, and real-time gross primary productivity product(2000-present) covering the Contiguous United States. https://www.earth-systsci-data-discuss.net/essd-2020-36/ (2020) doi:10.5194/essd-2020-36.

39. Badgley, G., Anderegg, L. D. L., Berry, J. A. \& Field, C. B. Terrestrial gross primary production: Using NIRv to scale from site to globe. Glob. Change Biol. 25, 3731-3740 (2019).

40. Wu, G. et al. Radiance-based NIR as a proxy for GPP of corn and soybean. Environ. Res. Lett. (2019) doi:10.1088/1748-9326/ab65cc.

41. Liu, L. et al. Estimating Maize GPP using Near-infrared Radiance of Vegetation. Sci. Remote Sens. 100009 (2020) doi:10.1016/j.srs.2020.100009.

42. Zhang, Z. et al. The potential of satellite FPAR product for GPP estimation: An indirect evaluation using solar-induced chlorophyll fluorescence. Remote Sens. Environ. 240, 111686 (2020).

43. Zhang, Y., Joiner, J., Alemohammad, S. H., Zhou, S. \& Gentine, P. A global spatially Continuous Solar Induced Fluorescence (CSIF) dataset using neural networks. Biogeosciences Discuss. 1-34 (2018) doi:10.5194/bg-2018-255.

44. Jung, M. et al. The FLUXCOM ensemble of global land-atmosphere energy fluxes. ArXiv Prepr. ArXiv181204951 (2018).

45. Jung, M. et al. Scaling carbon fluxes from eddy covariance sites to globe: synthesis and evaluation of the FLUXCOM approach. Biogeosciences 17, 1343-1365 (2020).

46. Guanter, L. et al. Global and time-resolved monitoring of crop photosynthesis with chlorophyll fluorescence. Proc. Natl. Acad. Sci. 111, E1327-E1333 (2014).

47. Wen, J. et al. A framework for harmonizing multiple satellite instruments to generate a long-term global high spatial-resolution solar-induced chlorophyll fluorescence (SIF). Remote Sens. Environ. 239, 111644 (2020). 
48. Drusch, M. et al. The FLuorescence EXplorer Mission Concept-ESA's Earth Explorer 8. IEEE Trans. Geosci. Remote Sens. 55, 1273-1284 (2017).

49. Lee, J.-E. et al. Simulations of chlorophyll fluorescence incorporated into the Community Land Model version 4. Glob. Change Biol. 21, 3469-3477 (2015).

50. Zhang, Y. et al. Estimation of vegetation photosynthetic capacity from space-based measurements of chlorophyll fluorescence for terrestrial biosphere models. Glob. Change Biol. 20, 3727-3742 (2014).

51. Camino, C., Gonzalez-Dugo, V., Hernandez, P. \& Zarco-Tejada, P. J. Radiative transfer Vcmax estimation from hyperspectral imagery and SIF retrievals to assess photosynthetic performance in rainfed and irrigated plant phenotyping trials. Remote Sens. Environ. 111186 (2019) doi:10.1016/j.rse.2019.05.005.

52. Croft, H. et al. The global distribution of leaf chlorophyll content. Remote Sens. Environ. 236, 111479 (2020).

53. Croft, H. et al. Leaf chlorophyll content as a proxy for leaf photosynthetic capacity. Glob. Change Biol. (2016) doi:10.1111/gcb.13599.

54. Gitelson, A. A. \& Gamon, J. A. The need for a common basis for defining light-use efficiency: Implications for productivity estimation. Remote Sens. Environ. 156, 196-201 (2015).

55. Ryu, Y., Jiang, C., Kobayashi, H. \& Detto, M. MODIS-derived global land products of shortwave radiation and diffuse and total photosynthetically active radiation at $5 \mathrm{~km}$ resolution from 2000 . Remote Sens. Environ. 204, 812-825 (2018).

56. Hao, D. et al. Estimating hourly land surface downward shortwave and photosynthetically active radiation from DSCOVR/EPIC observations. Remote Sens. Environ. 232, 111320 (2019).

57. Turner, A. J. et al. A double peak in the seasonality of California's photosynthesis as observed from space. https://www.biogeosciences-discuss.net/bg-2019-387/ (2019) doi:10.5194/bg-2019-387. 
58. Wohlfahrt, G. et al. Sun-induced fluorescence and gross primary productivity during a heat wave. Sci. Rep. 8, (2018).

59. Somkuti, P. et al. A new space-borne perspective of crop productivity variations over the US Corn Belt. Agric. For. Meteorol. 281, 107826 (2020).

60. Siegmann, B. The high-performance airborne imaging spectrometer HyPlant - From raw images to top-of-canopy reflectance and fluorescence products_An example dataset from the agricultural researchstation Campus Klein-Altendorf. (2019) doi:10.5447/IPK/2019/21.

61. Cogliati, S. et al. A Spectral Fitting Algorithm to Retrieve the Fluorescence Spectrum from Canopy Radiance. Remote Sens. 11, 1840 (2019).

62. Tucker, C. J. Red and photographic infrared linear combinations for monitoring vegetation. Remote Sens. Environ. 8, 127-150 (1979).

63. Rouse, J. W., Haas, R. H., Schell, J. A. \& Deering, D. W. Monitoring vegetation systems in the Great Plains with ERTS. Third ERTS Symp. NASA SP-351 1, 309-317 (1974).

64. Jiang, Z., Huete, A., Didan, K. \& Miura, T. Development of a two-band enhanced vegetation index without a blue band. Remote Sens. Environ. 112, 3833-3845 (2008).

65. Li, Z. et al. Solar-induced chlorophyll fluorescence and its link to canopy photosynthesis in maize from continuous ground measurements. Remote Sens. Environ. 236, 111420 (2020).

66. Goulas, Y. et al. Gross Primary Production of a Wheat Canopy Relates Stronger to Far Red Than to Red Solar-Induced Chlorophyll Fluorescence. Remote Sens. 9, 97 (2017).

67. Zhang, Z. et al. Reduction of structural impacts and distinction of photosynthetic pathways in a global estimation of GPP from space-borne solar-induced chlorophyll fluorescence. Remote Sens. Environ. 240, 111722 (2020).

68. Martini et al. Nitrogen and Phosphorus effect on Sun-Induced Fluorescence and Gross Primary Productivity in Mediterranean Grassland. Remote Sens. 11, 2562 (2019). 
69. Daumard, F. et al. A Field Platform for Continuous Measurement of Canopy Fluorescence. IEEE Trans. Geosci. Remote Sens. 48, 3358-3368 (2010).

70. Yang, X. et al. FluoSpec 2-An Automated Field Spectroscopy System to Monitor Canopy SolarInduced Fluorescence. 18 (2018).

71. Kim, J., Ryu, Y., Jiang, C. \& Hwang, Y. Continuous observation of vegetation canopy dynamics using an integrated low-cost, near-surface remote sensing system. Agric. For. Meteorol. 264, 164-177 (2019).

72. van der Tol, C., Verhoef, W., Timmermans, J., Verhoef, A. \& Su, Z. An integrated model of soilcanopy spectral radiances, photosynthesis, fluorescence, temperature and energy balance. Biogeosciences 6, 3109-3129 (2009).

73. Vilfan, N., van der Tol, C., Muller, O., Rascher, U. \& Verhoef, W. Fluspect-B: A model for leaf fluorescence, reflectance and transmittance spectra. Remote Sens. Environ. 186, 596-615 (2016).

74. Rascher, U. et al. Sun-induced fluorescence - a new probe of photosynthesis: First maps from the imaging spectrometer HyPlant. Glob. Change Biol. 21, 4673-4684 (2015).

75. Siegmann, B. et al. The High-Performance Airborne Imaging Spectrometer HyPlant-From Raw Images to Top-of-Canopy Reflectance and Fluorescence Products: Introduction of an Automatized Processing Chain. Remote Sens. 11, 2760 (2019).

76. Köhler, P. et al. Global Retrievals of Solar-Induced Chlorophyll Fluorescence With TROPOMI: First Results and Intersensor Comparison to OCO-2. Geophys. Res. Lett. (2018) doi:10.1029/2018GL079031.

77. Guanter, L. et al. Using field spectroscopy to assess the potential of statistical approaches for the retrieval of sun-induced chlorophyll fluorescence from ground and space. Remote Sens. Environ. 133, 52-61 (2013).

78. Siddans, R. S5p-NPP cloud processor ATBD. Rutherford Appleton Lab. Didcot UK (2017). 
79. Duveiller, G. et al. A spatially downscaled sun-induced fluorescence global product forenhanced monitoring of vegetation productivity. Earth Syst. Sci. Data Discuss. 1-24 (2019) doi:10.5194/essd2019-121.

80. Joiner, J. et al. Global monitoring of terrestrial chlorophyll fluorescence from moderate-spectralresolution near-infrared satellite measurements: methodology, simulations, and application to GOME-2. Atmospheric Meas. Tech. 6, 2803-2823 (2013). 